Preprint typeset using $\mathrm{LAT}_{\mathrm{E}} \mathrm{X}$ style emulateapj v. 11/10/09

\title{
FIVE GROUPS OF RED GIANTS WITH DISTINCT CHEMICAL COMPOSITION IN THE GLOBULAR CLUSTER NGC $2808^{1}$
}

\author{
Eugenio CARretta ${ }^{2}$
}

\begin{abstract}
The chemical composition of multiple populations in the massive globular cluster (GC) NGC 2808 is addressed with the homogeneous abundance re-analysis of 140 red giant branch (RGB) stars. UVES spectra for 31 stars and GIRAFFE spectra for the other giants were analysed with the same procedures used for about 2500 giants in $23 \mathrm{GCs}$ in our FLAMES survey, deriving abundances of Fe, O, Na, Mg, $\mathrm{Si}, \mathrm{Ca}, \mathrm{Ti}, \mathrm{Sc}, \mathrm{Cr}, \mathrm{Mn}$, and Ni. Iron, elements from $\alpha$-capture, and in the Fe-group do not show intrinsic scatter. On our UVES scale the metallicity of NGC 2808 is $[\mathrm{Fe} / \mathrm{H}]=-1.129 \pm 0.005 \pm 0.034$ ( \pm statistical \pm systematic error) with $\sigma=0.030$ (31 stars). Main features related to proton-capture elements are retrieved, but the improved statistics and the smaller associated internal errors allow to uncover five distinct groups of stars along the $\mathrm{Na}-\mathrm{O}$ anticorrelation. We observe large depletions in $\mathrm{Mg}$, anticorrelated with enhancements of $\mathrm{Na}$ and also $\mathrm{Si}$, suggestive of unusually high temperatures for proton-captures. About $14 \%$ of our sample is formed by giants with solar or subsolar $[\mathrm{Mg} / \mathrm{Fe}]$ ratios. Using the $[\mathrm{Na} / \mathrm{Mg}]$ ratios we confirm the presence of five populations with different chemical composition, that we called P1, P2, I1, I2, and $\mathrm{E}$ in order of decreasing $\mathrm{Mg}$ and increasing $\mathrm{Na}$ abundances. Statistical tests show that the mean ratios in any pair of groups cannot be extracted from the same parent distribution. The overlap with the five populations recently detected from UV photometry is good but not perfect, confirming that more distinct components probably exist in this complex GC.

Subject headings: Stars: abundances - Stars: atmospheres - Stars: Population II - Galaxy: globular clusters - Galaxy: globular clusters: individual: NGC 2808
\end{abstract}

\section{INTRODUCTION}

Galactic globular clusters (GCs) host multiple stellar populations of widely (sometime wildly) different chemical composition and slightly different ages, as shown since longtime by spectroscopy and more recently by photometric studies (see the review by Gratton, Carretta, Bragaglia 2012 for a summary of observations, references and topics on GCs).

The first evidence from abundance analyses in the last two decades of the past century revealed clear C$\mathrm{N}$, Na-O anticorrelations, as well as the first correlations between $\mathrm{Na}$ and $\mathrm{Al}$ abundances in a few GCs, reviewed by e.g. Smith (1987) and Kraft (1994). Very different abundances of these light elements in coeval stars at the same evolutionary stage in otherwise monometallic stellar aggregates did negate the very same definition of simple stellar population. However this concept was not abandoned until two milestones were reached. First, theory provided the understanding that the observed abundance patterns could be generated by the simultaneous action of the $\mathrm{NaNa}$ and $\mathrm{MgAl}$ cycles in the same stratification where the $\mathrm{ON}$ part of the CNO cycle is operating in H-buning at high temperature (e.g. Denisenkov and Denisenkova 1989; Langer et al. 1993). Second, the first observation of the $\mathrm{Na}-\mathrm{O}$ and $\mathrm{Mg}-\mathrm{Al}$ anticorrelations among unevolved GC stars (Gratton et al. 2001) unambigously demonstrated that the $\mathrm{Na}, \mathrm{Al}$-enhanced, O,Mg-depleted com-

\footnotetext{
${ }^{1}$ Based on data collected at the ESO telescopes under programme 072.D-0507 and during the FLAMES Science Verification programme.

2 INAF, Osservatorio Astronomico di Bologna, via Ranzani 1, 40127, Bologna, Italy. eugenio.carretta@oabo.inaf.it
}

position must be inherited by the most massive stars of a (several) previous stellar generation(s).

Since then, the (anti)correlations among protoncapture elements have been understood to represent the true DNA of GCs. These relations distinguish cluster stars from the other phylum of Pop.II field stars where only the basic evolutionary effects - first dredge-up and a second mixing episode after the red giant branch (RGB) bump in the luminosity function - on the lightest $\mathrm{C}$ and $\mathrm{N}$ elements are observed (Gratton et al. 2000). The best studied chemical signature is the Na-O anticorrelation, discovered by the Lick-Texas group (see Kraft 1994; Sneden 2000) and so widespread that can be considered as the fingerprint of a bona fide globular cluster (Carretta et al. 2010a). The ubiquity of this important tracer shows that almost all GCs are composed of multiple populations.

The more recent photometric approach to multiple populations in GCs exploits the presence in the filter passbands of absorption features of proton-capture elements. In most cases only the lightest elements $\mathrm{C}, \mathrm{N}$ are involved, with impact on those blue/near-UV filters including NH, CN, and CH features (e.g. Grundahl et al. 1998; Marino et al. 2008; Han et al. 2009; Lee et al. 2009; Monelli et al. 2013; Milone et al. 2012a, and references therein). Using pseudo-color indexes, where stars of different generations are more or less separated owing the different chemical composition, it is at present routinely possible to uncover broad or split photometric sequences in most GCs. The recent addition of the F275W passband of the WFC3 camera (Piotto et al. 2015), which samples an $\mathrm{OH}$ molecular band, is useful to connect these photometric sequences with heav- 
ier proton-capture elements like $\mathrm{O}$ (and $\mathrm{Na}$, through the NeNa and ON cycles). Although no photometric filter seems to be presently able to detect differences in the heaviest elements involved in proton-capture reactions (Mg, Al, Si), space based UV photometry confirms that most GCs are formed by multiple populations.

However, some GCs are more multiple than others. The most recent case is NGC 2808 where Milone et al. (2015) separated five distinct populations using a combination of F275W, F336W, and F438W filters from $H S T$. NGC 2808 is a pivotal object to investigate in detail the complex scenario of the origin of multiple populations in GCs, exploiting the wealth of photometric and spectroscopic data gathered for this cluster. Apart from $\omega$ Cen and M 54, whose metallicity spread tells a tale of a different origin (probably in/as a core of a former dwarf nucleated galaxy), NGC 2808 is the fifth more massive object in the catalogue by Harris (1996) of Galactic GCs $\left(M_{V}=-9.39\right)$. The present day total mass is found to be the driving parameter in establishing the extent of chemical differences among multiple populations (Carretta et al. 2010a). Moreover, the cluster mass is one of the more relevant factors (together with the metallicity) in determining whether proton-capture reactions are able to proceed to temperatures so high to significantly affect the primordial level of heavier elements like $\mathrm{Mg}$, from type II supernovae (Carretta et al. 2009a).

Three distinct main sequences (MS) were known since long time to exist in this GC (Piotto et al. 2007; Milone et al. 2012b), one of the best examples of the impact on the colour-magnitude diagrams (CMDs) of helium, the main outcome of the H-burning at high temperature. The distribution of stars along its horizontal branch (HB) is multimodal (Bedin et al. 2000) and reach quite hot effective temperature, again a good signature of enhanced He abundance in a fraction of stars.

Our group is actively working since many years on the chemical composition of stars in NGC 2808, gathering an unprecedented wealth of data. The first evidence of multiple populations in this cluster came from the analysis of $\mathrm{Na}$ abundances in 81 giants from moderately high resolution FLAMES spectra (Carretta et al. 2003). The spread in $[\mathrm{Na} / \mathrm{Fe}]^{3}$ was found to span a range of about 1 dex similar to what found in other GCs by the LickTexas group. Soon after, Carretta et al. 2004) confirmed the multiple populations in NGC 2808 by finding an extended $\mathrm{Na}-\mathrm{O}$ anticorrelation on the RGB from high resolution UVES spectra of 20 stars, with [O/Fe] ratios as low as -1 dex. This is not surprising, since there is a tight correlation between the extent of the Na-O anticorrelation and the maximum temperature along the HB (Carretta et al. 2007a), both features driven again by the same factor, He. NGC 2808 was one of the GCs used by Carretta (2006) to define the interquartile range of the $[\mathrm{O} / \mathrm{Na}]$ ratio, $\mathrm{IQR}[\mathrm{O} / \mathrm{Na}]$, as an efficient way to quantify the extension of the $\mathrm{Na}-\mathrm{O}$ anticorrelation and the relevance at large of changes in chemical composition due to the process of formation of a GC and its multiple population. Abundance analysis of horizontal branch (HB) stars was presented by Gratton et al. (2011) and

\footnotetext{
3 We adopt the usual spectroscopic notation, i.e. $[\mathrm{X}]=$ $\log (\mathrm{X})_{\text {star }}-\log (\mathrm{X}) \odot$ for any abundance quantity $\mathrm{X}$, and $\log \epsilon(\mathrm{X})$ $=\log \left(\mathrm{N}_{\mathrm{X}} / \mathrm{N}_{\mathrm{H}}\right)+12.0$ for absolute number density abundances.
}

Marino et al. (2014).

The analysis of GIRAFFE spectra for 120 giants in NGC 2808 (Carretta et al. 2006) started our FLAMES survey devoted to study the link between $\mathrm{Na}-\mathrm{O}$ anticorrelation and $\mathrm{HB}$ in about $25 \mathrm{GCs}$ (Carretta et al. 2006, 2007b, c, d, 2009a, b, 2010b, 2011, 2013a, 2014, 2015; Gratton et al. 2006, 2007). However, being the first target considered in the survey, NGC 2808 was analysed with slightly different procedures from those used for the other GCs, in particular the scale of effective temperatures, efficiently tuned for all other GCs to minimize the impact of uncertainties in the atmospheric parameters on star-to-star errors in abundances. This unconsistency calls for a re-analysis of all the available material to derive the chemical composition of stars in NGC 2808 in homogeneous way with respect to the other GCs in the survey.

First results of this re-analysis concerning the $\mathrm{Al}$ abundances (only available for 31 RGB stars with UVES spectra) were already presented in Carretta (2014) along with $\mathrm{Mg}$ abundances. The stars were found clustered into three discrete groups with different chemical composition along the $\mathrm{Mg}-\mathrm{Al}$ anticorrelation. The fractions of stars in the three components were found in excellent agreement with the number ratios computed by Milone et al. (2012b) on the three MSs.

In the present paper we provide the full derivation of the atmospheric parameters for all stars with available spectra from previous analyses. For 31 giants with UVES datat we derived abundances of $\mathrm{Fe}, \mathrm{O}, \mathrm{Na}, \mathrm{Si}, \mathrm{Ca}, \mathrm{Ti}$, $\mathrm{Sc}, \mathrm{Cr}, \mathrm{Mn}$, Ni. Homogeneous abundances of Fe, O, $\mathrm{Na}$ are derived again for the 123 giants with GIRAFFE spectra and new abundances of $\mathrm{Mg}$, Si, Ca, Ti, Sc, Cr, and $\mathrm{Ni}$ were obtained for the first time for this large set of stars. Homogeneous abundance ratios in our total sample extend the analysis of discrete components along the RGB of NGC 2808.

The paper is organized as follows: in $\S 2$ we describe the analysis method and the derivation of the atmospheric parameters with associated errors, the results for abundances, the $\mathrm{Na}-\mathrm{O}$ and $\mathrm{Na}-\mathrm{Mg}$ anticorrelations are presented in $\S 3$, whereas the chemical tagging of multiple populations in NGC 2808 is discussed in $\S 4$. Finally, in $\S 5$ we summarise our findings.

\section{ANALYSIS \\ 2.1. Sample}

The observational material of the present re-analysis consists in UVES spectra for 31 red giants and GIRAFFE spectra for 123 giants, all acquired with the FLAMES instrument (Pasquini et al. 2002) at the ESO VLT-UT2 telescope. We used proprietary UVES data (ESO Programme 072.D-0507) for 12 stars analysed in Carretta et al. (2009a) and UVES spectra from the FLAMES Science Verification for 19 stars whose analysis was done in Carretta et al. (2004) and Carretta (2006). These spectra have a resolution of $\mathrm{R} \sim 47,000$ and a spectral range between 4800 and $6800 \AA$, with a small gap at about $5900 \AA$. Coordinates and magnitudes for all 31 stars of the UVES sample can be found in Carretta (2014).

The GIRAFFE spectra were taken with the high resolution grating and two setups, HR11 ( $R=24100$, wave- 


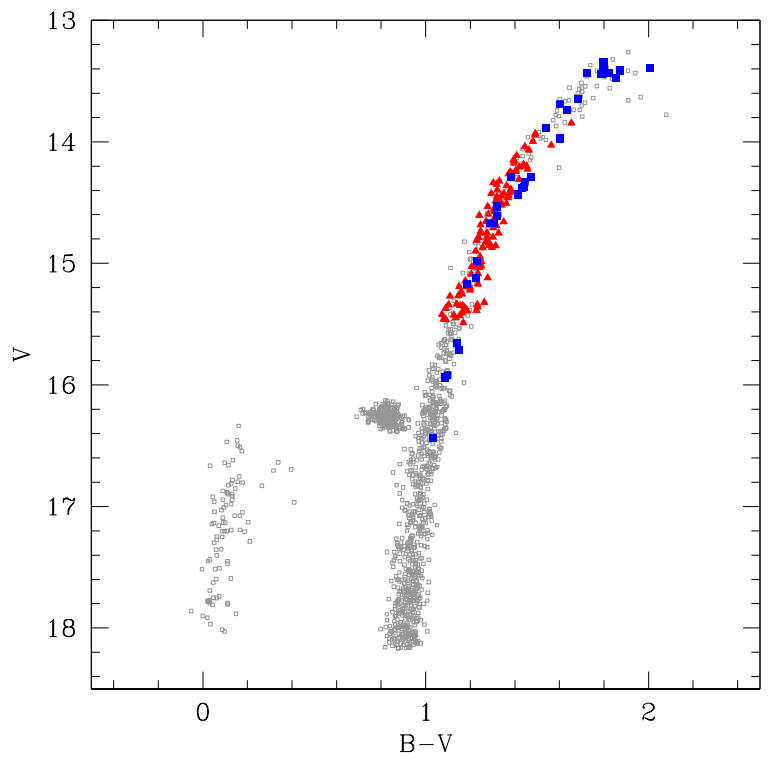

Figure 1. $V, B-V$ CMD of NGC 2808 (Bedin et al. 2000, small squares). Stars in the present sample observed with UVES are indicated with large blue squares, giants observed with GIRAFFE are represented by large red triangles.

length range 5597-5840 $\AA$ ) and $\operatorname{HR} 13$ ( $R=21900$, wavelength range $6120-6405 \AA$ ). In this GIRAFFE sample, 32 stars were observed with HR11 only, 28 with HR13 only and 63 have observations with both setups. Coordinates and magnitudes for these giants are in Carretta et al. (2006). Details of the observations and data reduction are reported in the original papers.

Fourteen out of 154 RGB stars were observed with both UVES and GIRAFFE, hence our final sample consists of 140 individual stars, all members of the RGB of NGC 2808 (see the original papers by Carretta et al. 2004, 2006), spanning the magnitude range $V=13.34 \div$ 16.44. In Fig. 1 the location of these RGB stars on the $V, B-V$ CMD from Bedin et al. (2000) is shown. Only one star is fainter than the RGB-bump level $V=16.235$ (Nataf et al. 2011). The optical photometry was integrated with $K$ band magnitudes from the Point Source Catalogue of 2MASS (Skrutskie et al. 2006) to derive atmospheric parameters as described in the next Section.

\subsection{Atmospheric parameters and metallicity}

All the abundances derived in the present re-analysis rest on equivalent widths $(E W \mathrm{~s})$ measured with the procedure described in Bragaglia et al. (2001) and using the latest version of the package ROSA Gratton 1988). A first difference with the original analysis in Carretta et al. (2006) is that this time the $E W$ s measured on the GIRAFFE spectra were corrected to the system defined by $E W$ s from the higher resolution UVES spectra. We used a linear regression between the two sets of measurements derived using 478 lines in common for the 14 stars observed with both instruments. The corrected $E W$ s from GIRAFFE are plotted as a function of the UVES $E W$ s in Fig 2 .

This is the first step to make the present analysis perfectly consistent with the abundance analysis of more than 2500 RGB stars in the other GCs observed in our FLAMES survey.

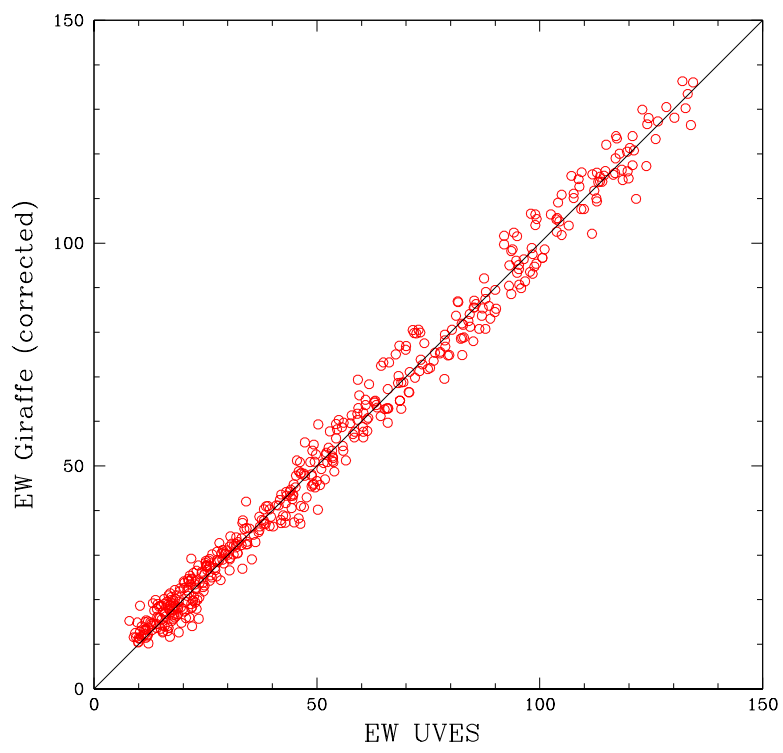

Figure 2. $E W$ s measured on GIRAFFE spectra, after the correction to the UVES systems, as a function of the $E W \mathrm{~s}$ measured on UVES spectra for the 14 giants observed with both spectrographs.

The second step concerns the effective temperature $\left(\mathrm{T}_{\text {eff }}\right)$ scale. In Carretta et al. (2004, 2006) and Carretta (2006) the giants of our sample were analysed using $\mathrm{T}_{\text {eff }}$ values derived from $V-K$ colours and the calibration by Alonso et al. (1999, 2001). The new approach followed for the other $23 \mathrm{GCs}$ and adopted here also for NGC 2808 is to use the above temperatures as first pass values: the final values adopted for all our target stars were obtained using an average relation between $\mathrm{T}_{\text {eff }}(V-K)$ and the $K$ apparent magnitudes of the RGB stars. This adopted procedure has three advantages: it decreases the star-to-star scatter in abundances due to uncertainties in temperatures (magnitudes are more precise than colours), it minimises the impact of the differential reddening in NGC 2808 on derived $\mathrm{T}_{\text {eff }}$, and finally it is fully consistent with the procedure adopted for all the other GCs.

Surface gravities $\log g$ were obtained from bolometric corrections (from Alonso et al.), the newly derived effective temperatures, reddening and distance modulus from Harris (1996), and assuming masses of $0.85 \mathrm{M}_{\odot}$ and $M_{\mathrm{bol}, \odot}=4.75$ as bolometric magnitude for the Sun.

Finally, values of the microturbulent velocity $v_{t}$ were obtained by eliminating in each star trends of the abundances from $\mathrm{Fe}$ I lines with the expected line strength (see Magain 1984). Final abundances were derived from $E W$ analysis, by choosing the model in the Kurucz (1993) grid of solar-scaled LTE model atmospheres with the overshooting option switched on, with the proper atmospheric parameters, whose abundance matched the one derived from $\mathrm{Fe}$ I lines. Adopted line list, atomic parameters and solar reference abundances are described in Gratton et al. (2003).

The new, final atmospheric parameters are listed with the derived Fe abundances of the 140 individual stars in Tab. 1]

NGC 2808 is confirmed to be monometallic, as far the content of iron is concerned. On the homoge- 


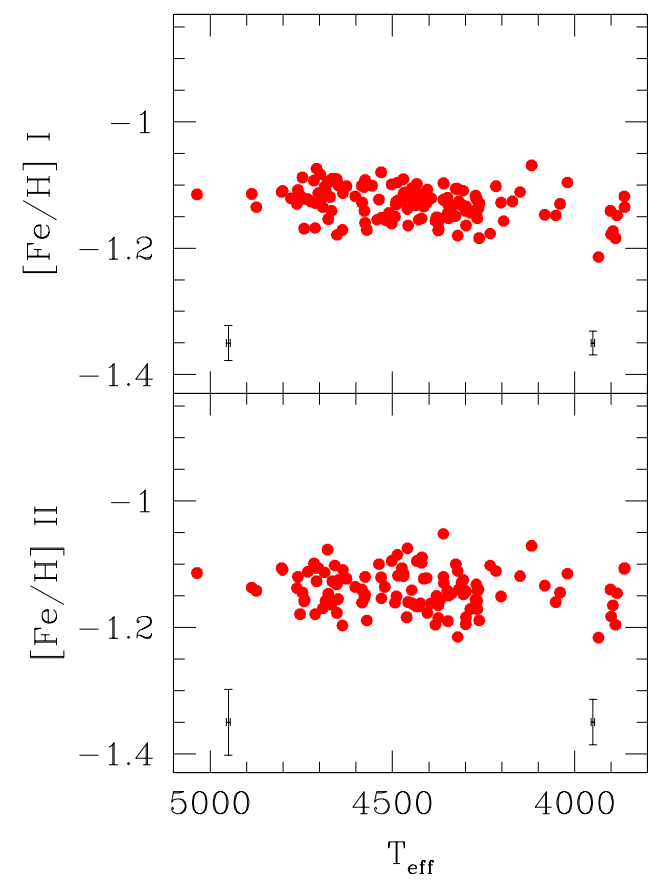

Figure 3. Abundance ratios $[\mathrm{Fe} / \mathrm{H}]$ I (upper panel) and $[\mathrm{Fe} / \mathrm{H}]$ II (lower panel) as a function of $\mathrm{T}_{\text {eff }}$ for all $140 \mathrm{RGB}$ stars in the present re-analysis. Error bars on the right and on the left side are star-to-star errors for targets observed with UVES and GIRAFFE, respectively.

neous metallicity scale based on high resolution UVES spectra (Carretta et al. 2009c) the metal abundance in NGC 2808 is on average $[\mathrm{Fe} / \mathrm{H}] \mathrm{I}=-1.129 \pm 0.005 \pm 0.034$ $\operatorname{dex}(\sigma=0.030)$ from the 31 stars observed with UVES (where the first and second error bars refer to the statistical and systematic errors, respectively, see next Section). From the reanalysis of the 123 stars with GIRAFFE spectra we derive on average $[\mathrm{Fe} / \mathrm{H}] \mathrm{I}=-1.128 \pm 0.002 \pm 0.046$ $\operatorname{dex}(\sigma=0.026)$. We then are entitled to merge the two subsample, adopting the values from UVES for all abundances in the 14 stars observed with both instruments. The improvement due to the new homogeneous procedure is immediately evident in the decrease of the $\mathrm{rms}$ scatters associated to the mean Fe values, the previous values being $\sigma=0.075$ and $\sigma=0.065$ for UVES and GIRAFFE, respectively.

Abundances of iron derived from singly ionized transitions are in very good agreement with the above values. We derived $[\mathrm{Fe} / \mathrm{H}] \mathrm{II}=-1.128 \operatorname{dex}(\sigma=0.026 \operatorname{dex}, 31$ stars $)$ and $[\mathrm{Fe} / \mathrm{H}] \mathrm{II}=-1.143 \operatorname{dex}(\sigma=0.033 \operatorname{dex}, 91$ stars) from the UVES and GIRAFFE samples, respectively.

Derived abundances of $[\mathrm{Fe} / \mathrm{H}]$ I and $[\mathrm{Fe} / \mathrm{H}]$ II are plotted as a function of the effective temperatures in Fig. 3. together with internal, star-to-star error bars as estimated in the next subsection.

\subsection{Abundances and error analysis}

Abundances of Fe, O, Na, Mg, Al, Si, Ca, Ti, Cr, $\mathrm{Mn}$, and Ni for 19 giants with UVES spectra from the FLAMES Science Verification were originally presented in Carretta et al. (2004) and Carretta (2006). O, Na, $\mathrm{Mg}, \mathrm{Al}$, and Si for the 12 RGB stars observed with UVES
Table 2

Mean abundances from UVES and GIRAFFE

\begin{tabular}{|c|c|c|c|c|c|}
\hline \multirow[t]{2}{*}{ lement } & \multicolumn{3}{|c|}{ UVES } & \multicolumn{2}{|c|}{ GIRAFFE } \\
\hline & $\mathrm{n}$ & avg & $\mathrm{rms}$ & $\mathrm{n} \quad$ avg & $\mathrm{rms}$ \\
\hline$[\mathrm{O} / \mathrm{Fe}] \mathrm{I}$ & 31 & +0.06 & 0.34 & $91+0.00$ & 0.38 \\
\hline$[\mathrm{Na} / \mathrm{Fe}]_{\mathrm{I}}$ & 31 & +0.20 & 0.20 & $123+0.2$ & 0.23 \\
\hline$[\mathrm{Mg} / \mathrm{Fe}] \mathrm{I}$ & 31 & +0.26 & 0.16 & $122+0.2$ & 0.16 \\
\hline$[\mathrm{Al} / \mathrm{Fe}] \mathrm{I}$ & 31 & +0.46 & 0.47 & & \\
\hline$[\mathrm{Si} / \mathrm{Fe}] \mathrm{I}$ & 31 & $+0.2 s$ & 0.07 & $123+0.3$ & 0.05 \\
\hline$[\mathrm{Ca} / \mathrm{Fe}] \mathrm{I}$ & 31 & +0.3 & 0.02 & $123+0.3$ & 0.03 \\
\hline$[\mathrm{Sc} / \mathrm{Fe}] \mathrm{II}$ & 31 & -0.01 & 0.04 & $123-0.00$ & 0.05 \\
\hline$[\mathrm{Ti} / \mathrm{Fe}]_{\mathrm{I}}$ & 31 & +0.21 & 0.02 & $123+0.2$ & 0.04 \\
\hline$[\mathrm{Ti} / \mathrm{Fe}] \mathrm{II}$ & 29 & +0.18 & 0.02 & & \\
\hline$[\mathrm{Cr} / \mathrm{Fe}] \mathrm{I}$ & 31 & -0.0 & 0.02 & $123-0.03$ & 0.03 \\
\hline$[\mathrm{Cr} / \mathrm{Fe}]_{\mathrm{II}}$ & 31 & -0.0 & 0.03 & & \\
\hline$[\mathrm{Mn} / \mathrm{Fe}] \mathrm{I}$ & 31 & -0.37 & 0.04 & & \\
\hline$[\mathrm{Fe} / \mathrm{H}]_{\mathrm{I}}$ & 31 & -1.13 & 0.03 & $123-1.13$ & 0.03 \\
\hline$[\mathrm{Fe} / \mathrm{H}] \mathrm{II}$ & 31 & -1.14 & 0.03 & $91-1.1$ & 0.03 \\
\hline$[\mathrm{Ni} / \mathrm{Fe}]_{\mathrm{I}}$ & 31 & -0.06 & 0.02 & $123-0.08$ & 0.02 \\
\hline
\end{tabular}

Note: individual abundances of $\mathrm{Al}$ are reported in Carretta (2014).

in our FLAMES survey were analysed originally with other 202 stars in 17 GCs in Carretta et al. (2009a). These abundances are derived again using the new scale of atmospheric parameters discussed above for all 31 stars of the UVES sample and are presented here for the first time, except for abundances of $\mathrm{Al}$ and $\mathrm{Mg}$ Carretta 2014). For these stars, abundances of $\mathrm{Ti}$ and $\mathrm{Cr}$ were obtained using both neutral and singly ionized species, with results in excellent agreement.

For the 123 stars in the GIRAFFE sample, Fe, $\mathrm{O}, \mathrm{Na}$ were revisited with respect to the values in Carretta et al. (2006) obtained with the previous temperature scale and without correcting the $E W$ s to the UVEs system. Abundances of $\mathrm{Mg}, \mathrm{Si}, \mathrm{Ca}, \mathrm{Ti}, \mathrm{Cr}, \mathrm{Ni}$ are obtained for the first time in the present analysis. Due to the limited spectral range of GIRAFFE spectra, only transitions of neutral $\mathrm{Ti}$ and $\mathrm{Cr}$ were available for this set of giants.

Abundances of $\mathrm{Na}$ were corrected for effects of departures from the LTE assumption using prescriptions by Gratton et al. (1999), whereas abundance corrections for Sc and Mn as in Gratton et al. (2003) were adopted to account for the hyperfine structure.

In summary, we obtained homogeneous abundances of the elements $\mathrm{O}, \mathrm{Na}, \mathrm{Mg}$, Si involved in proton-capture reactions. Moreover, we derived abundances of elements representative of the $\alpha$-capture process $(\mathrm{Ca}, \mathrm{Ti})$ and of the Fe-group ( $\mathrm{Sc}, \mathrm{Cr}, \mathrm{Mn}, \mathrm{Ni}$ ). Average abundance ratios and their r.m.s. scatter are listed in Tab. 2 where the average value of Al by Carretta (2014) is also indicated. The abundances are listed separately for the UVES and GIRAFFE sets, but whenever possible we adopted UVES abundances for individual stars in our merged sample.

The sensitivity of the derived abundances to variations in the adopted atmospheric parameters for each element was obtained by re-iterating the analysis and varying each time only one parameter of the amount shown in Tab. 3. For the final slopes of the relations between the variation in each parameter and the abundance we took the average over all stars. This computation was done separately for UVES and GIRAFFE.

These sensitivities were used to estimate the impact 


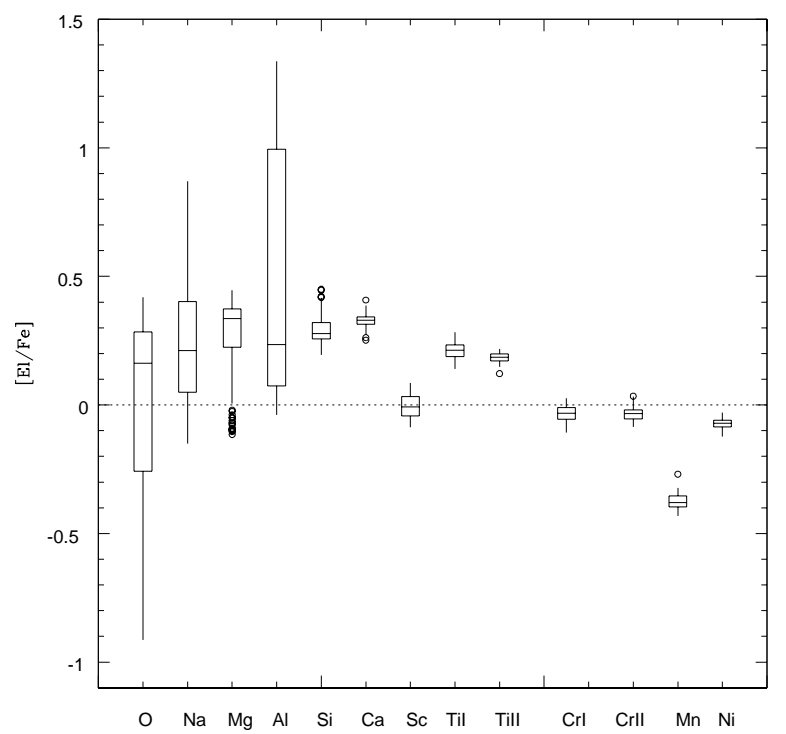

Figure 4. Box-and-whiskers plot of abundance ratios in RGB stars of NGC 2808. The horizontal line in each box is the median ratio of a given element. The bottom and the top lines of the box are the 25 th and the 75 th percentiles, whose distance (i.e. the box length) indicates the interquartile range, encompassing the middle $50 \%$ of the data. The vertical "whiskers" protruding from the box indicate the full abundance range excluding the outliers, defined as points lying more than 1.5 times the interquartile range from the 25 th or 75 th percentiles and indicated with open circles.

of uncertainties in atmospheric parameters on the derived abundances. A detailed description is given in Carretta et al. (2007a, 2009a) respectively for the analysis of GIRAFFE and UVES spectra. We note here that with respect to the analysis of NGC 2808 in Carretta et al. (2006, 2009a) the internal error in temperature is now reduced by almost an order of magnitude, from $44 \mathrm{~K}$ to $5 \mathrm{~K}$, with the presently adopted procedure for $\mathrm{T}_{\text {eff }}$. This improvement is very important to minimise the star-to-star errors in abundances (Tab. 3), a crucial step for a meaningful study of possible segregation of stars into groups with discrete chemical composition.

\section{RESULTS}

For individual stars, abundances of proton-capture elements are given in Tab. 4. In Tab. 5] are listed abundances of $\alpha=$ and Fe-group elements for species measured both on UVES and GIRAFFE spectra. Abundances of Ti II, Cr II and Mn (only available from UVES spectra) are listed in Tab. 6. The run of abundance ratios in NGC 2808 does not show any trend as a function of effective temperature.

We provide in Fig. 4 a graphical summary of the derived abundances in NGC 2808, using the so called boxand-whishers plot (Tukey 1977) which is particularly well suited to visualise possible skewed distributions and to investigate the presence of outstanding outliers.

The abundance pattern observed in NGC 2808 is typical of massive, normal GCs. Light elements involved in proton-capture reactions present a huge spread, exceeding many times the uncertainties associated to the analysis. Pure $\alpha$-process elements like $\mathrm{Ca}$ and $\mathrm{Ti}$ (not touched by proton-capture reactions, like instead $\mathrm{Mg}$ and $\mathrm{Si}$ ) show a much more limited range, with overabun-

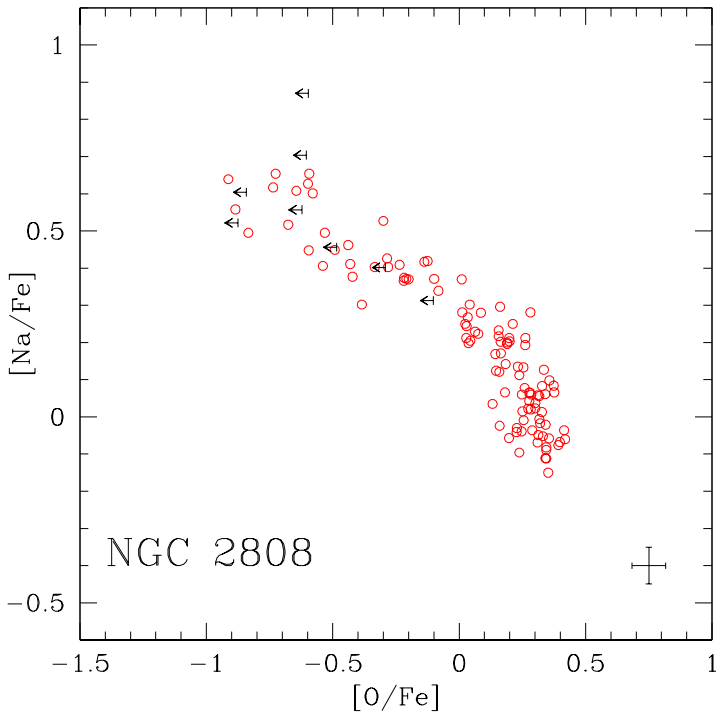

Figure 5. Anticorrelation of $\mathrm{O}$ and $\mathrm{Na}$ abundances in RGB stars of NGC 2808 from the present reanalysis. Arrow indicate upper limits in $[\mathrm{O} / \mathrm{Fe}]$. Star-to-star errors are those referred to the bulk of stars, observed with GIRAFFE. Internal errors for the 31 stars with UVES spectra are slighly smaller.

dances typical of normal nucleosynthesis from type II supernovae. Similarly, species belonging to the Fe-peak follow rather well the run of iron, apart from Mn which is underabundant as in normal halo stars of similar metallicity.

However, already from this compact representation it is immediately clear that Mg in NGC 2808 is outstanding even among proton-capture elements. The improved statistics of the present analysis, 140 RGB stars compared to the maximum of 31 stars with $\mathrm{Mg}$ abundance analysed in Carretta (2014), allows us to uncover an unusually large number of outliers in the $\mathrm{Mg}$ distribution, all of them with subsolar $[\mathrm{Mg} / \mathrm{Fe}]$ ratios. Also the Si distribution seems to be particular, with a number of outliers located at the high-abundance border of the distribution, i.e. anti-correlated with the behaviour observed in $\mathrm{Mg}$.

\subsection{The classical $\mathrm{Na-O}$ anticorrelation}

The updated version of the Na-O anticorrelation, the most outstanding typical signature of the chemical composition in a GC, is shown in Fig. 5. Abundances of $\mathrm{Na}$ are available for all 140 stars in our merged sample, because Na lines fall in both the HR11 and HR13 setups (and obviously in the UVES spectral range). However, abundances of $\mathrm{O}$ are only available for 91 stars observed with the GIRAFFE setup HR13; when merged with the UVES sample, our final sample includes 117 stars with both $\mathrm{O}$ and $\mathrm{Na}$, which is however the largest to date used to study this feature in a normal, monometallic GC.

The present re-analysis confirms many previous results (Carretta et al. 2006): the Na-O anticorrelation is very extended, showing stars with $\mathrm{O}$ abundances reaching almost $[\mathrm{O} / \mathrm{Fe}]=-1 \mathrm{dex}$. The interquartile range of the $[\mathrm{O} / \mathrm{Na}]$ ratio, $\mathrm{IQR}[\mathrm{O} / \mathrm{Na}]$, introduced by Carretta (2006) to provide a quantitative measure of changes in chemical composition between stars of different gener- 
Table 3

Sensitivities of abundance ratios to variations in the atmospheric parameters and to errors in the equivalent widths, and errors in abundances for stars of NGC 2808 observed with UVES and GIRAFFE

\begin{tabular}{|c|c|c|c|c|c|c|c|c|}
\hline \multicolumn{9}{|c|}{ UVES } \\
\hline Element & $\begin{array}{r}\text { Average } \\
\text { n. lines }\end{array}$ & $\begin{array}{l}T_{\text {eff }} \\
(K)\end{array}$ & $\begin{array}{r}\log g \\
(\operatorname{dex})\end{array}$ & $\begin{array}{l}{[\mathrm{A} / \mathrm{H}]} \\
(\mathrm{dex})\end{array}$ & $\begin{array}{r}v_{t} \\
\mathrm{kms}^{-1}\end{array}$ & $\begin{array}{l}\text { EWs } \\
\text { (dex) }\end{array}$ & $\begin{array}{r}\text { Total } \\
\text { Internal }\end{array}$ & $\begin{array}{r}\text { Total } \\
\text { Systematic }\end{array}$ \\
\hline Variation & & 50 & 0.20 & 0.10 & 0.10 & & & \\
\hline Internal & & 5 & 0.04 & 0.03 & 0.05 & 0.01 & & \\
\hline Systematic & & 46 & 0.06 & 0.03 & 0.01 & & & \\
\hline$[\mathrm{Fe} / \mathrm{H}] \mathrm{I}$ & 71 & +0.036 & +0.015 & +0.003 & -0.031 & 0.009 & 0.019 & 0.034 \\
\hline$[\mathrm{Fe} / \mathrm{H}]_{\mathrm{II}}$ & 9 & -0.055 & +0.102 & +0.031 & -0.015 & 0.027 & 0.036 & 0.059 \\
\hline$[\mathrm{O} / \mathrm{Fe}] \mathrm{I}$ & 2 & -0.022 & +0.067 & +0.029 & +0.028 & 0.057 & 0.061 & 0.066 \\
\hline$[\mathrm{Na} / \mathrm{Fe}] \mathrm{I}$ & 4 & +0.011 & -0.048 & -0.034 & +0.013 & 0.040 & 0.043 & 0.040 \\
\hline$[\mathrm{Mg} / \mathrm{Fe}]_{\mathrm{I}}$ & 3 & -0.009 & -0.013 & -0.006 & +0.016 & 0.046 & 0.047 & 0.029 \\
\hline$[\mathrm{Al} / \mathrm{Fe}] \mathrm{I}$ & 2 & +0.007 & -0.023 & -0.011 & +0.021 & 0.057 & 0.058 & 0.086 \\
\hline$[\mathrm{Si} / \mathrm{Fe}] \mathrm{I}$ & 7 & -0.050 & +0.020 & +0.008 & +0.021 & 0.030 & 0.033 & 0.048 \\
\hline$[\mathrm{Ca} / \mathrm{Fe}] \mathrm{I}$ & 15 & +0.027 & -0.031 & -0.015 & -0.017 & 0.021 & 0.024 & 0.027 \\
\hline$[\mathrm{Sc} / \mathrm{Fe}] \mathrm{II}$ & 8 & +0.048 & -0.023 & -0.004 & -0.016 & 0.028 & 0.030 & 0.045 \\
\hline$[\mathrm{Ti} / \mathrm{Fe}] \mathrm{I}$ & 9 & +0.051 & -0.019 & -0.016 & -0.005 & 0.027 & 0.028 & 0.047 \\
\hline$[\mathrm{Ti} / \mathrm{Fe}] \mathrm{II}$ & 9 & +0.041 & -0.030 & -0.010 & -0.024 & 0.027 & 0.030 & 0.039 \\
\hline$[\mathrm{Cr} / \mathrm{Fe}] \mathrm{I}$ & 17 & +0.036 & -0.030 & -0.015 & -0.007 & 0.019 & 0.021 & 0.035 \\
\hline$[\mathrm{Cr} / \mathrm{Fe}] \mathrm{II}$ & 10 & +0.016 & -0.026 & -0.017 & +0.004 & 0.025 & 0.026 & 0.018 \\
\hline$[\mathrm{Mn} / \mathrm{Fe}] \mathrm{I}$ & 3 & +0.016 & -0.012 & -0.007 & -0.015 & 0.046 & 0.047 & 0.016 \\
\hline$[\mathrm{Ni} / \mathrm{Fe}] \mathrm{I}$ & 26 & -0.011 & +0.015 & +0.006 & +0.010 & 0.016 & 0.017 & 0.012 \\
\hline \multicolumn{9}{|c|}{ GIRAFFE } \\
\hline Element & $\begin{array}{r}\text { Average } \\
n . \text { lines }\end{array}$ & $\begin{array}{l}T_{\text {eff }} \\
(\mathrm{K})\end{array}$ & $\begin{array}{l}\log g \\
(\operatorname{dex})\end{array}$ & $\begin{array}{l}{[\mathrm{A} / \mathrm{H}]} \\
(\mathrm{dex})\end{array}$ & $\begin{array}{r}v_{t} \\
\mathrm{kms}^{-1}\end{array}$ & $\begin{array}{c}\text { EWs } \\
\text { (dex) }\end{array}$ & $\begin{array}{r}\text { Total } \\
\text { Internal }\end{array}$ & $\begin{array}{r}\text { Total } \\
\text { Systematic }\end{array}$ \\
\hline Variation & & 50 & 0.20 & 0.10 & 0.10 & & & \\
\hline Internal & & 5 & 0.04 & 0.03 & 0.08 & 0.01 & & \\
\hline Systematic & & 46 & 0.06 & 0.05 & 0.01 & & & \\
\hline$[\mathrm{Fe} / \mathrm{H}] \mathrm{I}$ & 37 & +0.050 & +0.005 & -0.003 & -0.030 & 0.013 & 0.028 & 0.046 \\
\hline$[\mathrm{Fe} / \mathrm{H}] \mathrm{II}$ & 3 & -0.039 & +0.096 & +0.027 & -0.013 & 0.047 & 0.052 & 0.046 \\
\hline$[\mathrm{O} / \mathrm{Fe}] \mathrm{I}$ & 2 & -0.041 & +0.079 & +0.035 & +0.035 & 0.057 & 0.067 & 0.060 \\
\hline$[\mathrm{Na} / \mathrm{Fe}] \mathrm{I}$ & 3 & -0.004 & -0.037 & -0.019 & +0.016 & 0.047 & 0.049 & 0.024 \\
\hline$[\mathrm{Mg} / \mathrm{Fe}] \mathrm{I}$ & 2 & -0.014 & -0.009 & -0.004 & +0.016 & 0.057 & 0.058 & 0.020 \\
\hline$[\mathrm{Si} / \mathrm{Fe}] \mathrm{I}$ & 8 & -0.051 & +0.026 & +0.012 & +0.026 & 0.029 & 0.036 & 0.048 \\
\hline$[\mathrm{Ca} / \mathrm{Fe}] \mathrm{I}$ & 5 & +0.015 & -0.029 & -0.010 & -0.019 & 0.036 & 0.040 & 0.016 \\
\hline$[\mathrm{Sc} / \mathrm{Fe}] \mathrm{II}$ & 5 & -0.055 & +0.077 & +0.031 & +0.001 & 0.036 & 0.041 & 0.030 \\
\hline$[\mathrm{Ti} / \mathrm{Fe}] \mathrm{I}$ & 4 & +0.032 & -0.013 & -0.012 & +0.006 & 0.041 & 0.041 & 0.056 \\
\hline$[\mathrm{Cr} / \mathrm{Fe}] \mathrm{I}$ & 5 & +0.014 & -0.014 & -0.007 & +0.022 & 0.036 & 0.040 & 0.014 \\
\hline$[\mathrm{Ni} / \mathrm{Fe}] \mathrm{I}$ & 8 & -0.014 & +0.017 & +0.008 & +0.021 & 0.029 & 0.033 & 0.014 \\
\hline
\end{tabular}

ations, is 0.925 . This value, although slightly smaller than the old value (0.999), perfectly fits the relation with the cluster total present-day mass (as represented by the proxy of the total absolute magnitude $M_{V}$ ). In the same way, we confirm that NGC 2808 lies very well on the relation discovered by Carretta et al. (2007e) linking the extension of the Na-O anticorrelation with the HB morphology, as represented by the maximum temperature along the HB (Recio-Blanco et al. 2006).

The division of stars into the three components defined in Carretta et al. (2009b), primordial P and two components of second generation stars with intermediate $\mathrm{I}$ and extreme $\mathrm{E}$ composition, gives the fractions $\mathrm{P}=46 \pm 6 \%, \mathrm{I}=36 \pm 6 \%$, and $\mathrm{E}=18 \pm 4 \%$, where the attached error are from Poisson statistics. We confirm that NGC 2808 is one of the GCs where the highest fraction of first generation stars was retained: almost half of the present day stellar population belongs to the $\mathrm{P}$ component, whereas the observed mean over more than 20 GCs homogeneously analysed by our group is that only a third of stars show a pristine composition.

Second generation stars (component I and E) show a tendency to be more concentrated than stars with pri- mordial composition (Fig. 6): a two-tail KolmogorovSmirnov test allow us to safely reject the null hypothesis that the two distributions are extracted from the same parent population. However, due to the limitations imposed by the fiber positioning of FLAMES, only a small fraction of stars in our sample is located within two halmass radii from the cluster centre. Coupled to the different concentration of first and second generation stars this suggests that more ample photometric dataset are better suited for a more thorough study of this issue.

At first blush there seems to be not much difference with respect to previous results. However, the improved statistics of the merged sample and the decrease of internal errors due to the atmospheric parameters uncover a more subtle level of complexity in this cluster. The distribution function of the $[\mathrm{O} / \mathrm{Na}]$ ratio reveals that stars along the $\mathrm{Na}-\mathrm{O}$ anticorrelation seem to be splitted not simply in three, but into several structures, namely five groups peaked at about $[\mathrm{O} / \mathrm{Na}]=+0.30,0.0,-0.65,-0.9$, and -1.3 dex (see Fig. 7).

Let us assume, as a working hypothesis, that both the $\mathrm{P}$ and I components in NGC 2808 are composed each of two sub-groups. The separation of the last three peaks 


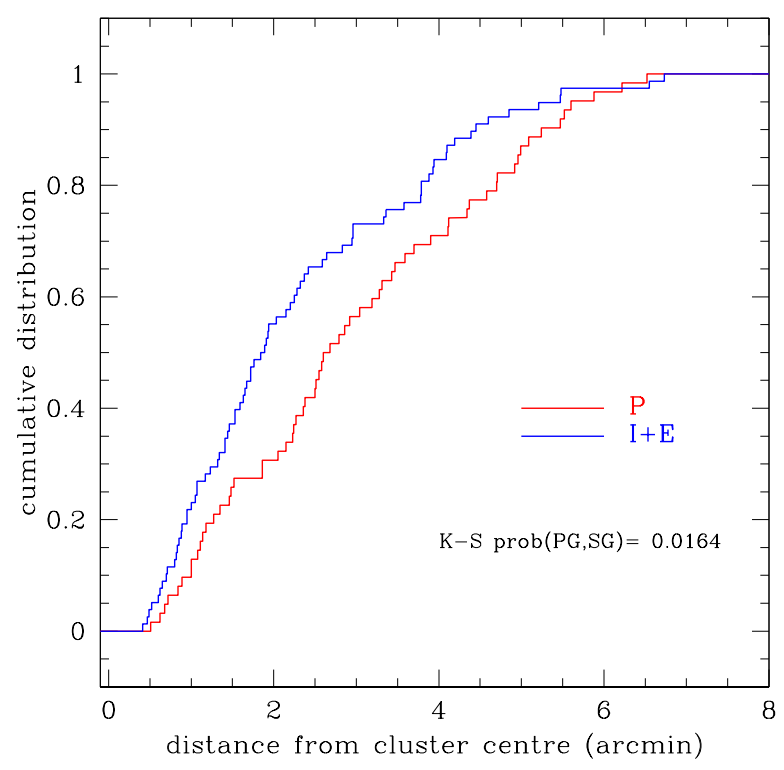

Figure 6. Cumulative distribution of radial distances from the cluster centre for stars of first ( $\mathrm{P}$ component) and of second $(\mathrm{I}+\mathrm{E})$ generation in NGC 2808. The probability of a KolmogorovSmirnov test is also listed.

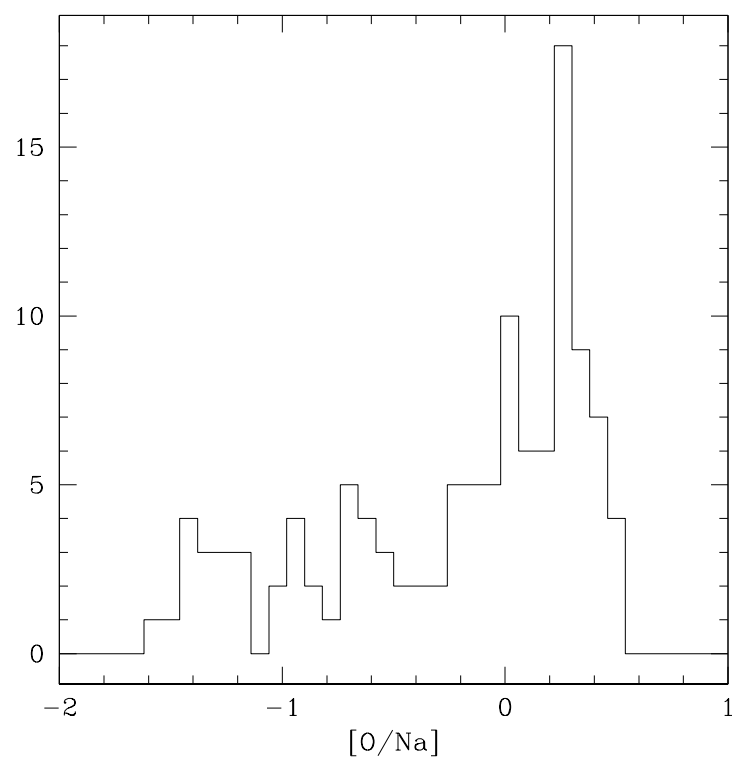

Figure 7. Distribution function of the $[\mathrm{O} / \mathrm{Na}]$ ratios of 117 giants in NGC 2808

at low $[\mathrm{O} / \mathrm{Na}]$ values in Fig. 7 is quite large, but even considering conservatively the larger errors estimated for GIRAFFE also the distance between the first two peaks exceeds by almost four times the combined internal uncertainty in $\mathrm{O}$ and $\mathrm{Na}$ (see Tab. 3). This means that the ensemble of stars with primordial composition in NGC 2808 is not a monolitic group, but rather composed by two sub-components, with small differences in the average $[\mathrm{O} / \mathrm{Fe}]$ ratio and larger differences in the $\mathrm{Na}$ content.

The existence of these two groups is in excellent agreement with the independent result obtained from the abundance analysis of HB stars in NGC 2808 by
Gratton et al. (2011). By observing several tens of stars they found some evidence of $\mathrm{Na}-\mathrm{O}$ anticorrelation already among the red $\mathrm{HB}$ (RHB) stars that should be the descendents of He-poor, O-rich stars. Gratton et al. (2011) concluded that the stellar populations in NGC 2808 must be more than three, since at least part of the red HB stars does not belong to the primordial cluster population as expected from the classical paradigm relating $\mathrm{O}$-rich stars to normal helium content. Recently, Marino et al. (2014), who did not derive O abundances for their sample of $\mathrm{HB}$ stars, confirmed a bimodality in Na among RHB stars in NGC 2808.

We may tentatively conclude that we found in our sample of $R G B$ stars the progenitors of the two groups that end up on the $\mathrm{RHB}$, with normal $\mathrm{He}$, similarly high $\mathrm{O}$, and slightly different $\mathrm{Na}$ content.

Two other groups seen in Fig. 7 present a composition typical of intermediate second generation stars, while the last is clearly identifiable with the extreme component as defined in Carretta et al. (2009b), characterised by very low $\mathrm{O}$ abundances and high $\mathrm{Na}$ values.

The coincidence of five groups distinct by their different chemical composition with five populations found photometrically by Milone et al. (2015) is a result beyond our expectations when this re-analysis was started simply to bring NGC 2808 onto the homogeneous system used for all other GCs in our FLAMES survey. Fortunately, we have now the possibility to investigate this finding in deeper details using abundances of other proton-capture elements as a sanity check, showing that there is not a simple correspondence between results from spectroscopy and photometry. The scenario in NGC 2808 looks more complex.

\subsection{The $\mathrm{Na}-\mathrm{Mg}$ anticorrelation confirms five discrete groups in $N G C 2808$}

Several reasons prompted us to use the relation between $\mathrm{Na}$ and $\mathrm{Mg}$ abundances to check the reality of the five chemically distinct components on the RGB of NGC 2808. These elements are the outcome of the two different cycles ( $\mathrm{NeNa}$ and $\mathrm{MgAl}$ ), hence they sample different reactions occurring at different temperatures, thus mass stratifications. Their interplay is then a good candidate to offer a panoramic view over all the mass range of possible polluters that contributed gas enriched in proton-capture elements at the epoch of cluster formation.

From a more practical point of view, all 140 giants in our final sample have $\mathrm{Na}$ abundances and we cannot derive a $\mathrm{Mg}$ abundance only for one star, with a net gain of about $20 \%$ in statistics with respect to the O-Na plot.

Abundances of $\mathrm{Na}$ and $\mathrm{Mg}$ are plotted in Fig. 8. The ratios $[\mathrm{Na} / \mathrm{Fe}]$ and $[\mathrm{Mg} / \mathrm{Fe}]$ in $\mathrm{RGB}$ stars of NGC 2808 are anticorrelated, with $\mathrm{Mg}$ decreasing by about 0.55 dex while Na increases by about 1 dex. A Spearman correlation test provides a probability that $\mathrm{Na}$ and $\mathrm{Mg}$ are not anticorrelated of less than $1.0 \times 10^{-6}$. This feature is not surprising, apart from the large fraction of stars with severe $\mathrm{Mg}$ depletions in this GC, being simply the consequence of $\mathrm{Na}$ production from ${ }^{22} \mathrm{Ne}$ with simultaneous $\mathrm{Mg}$ consumption from the $\mathrm{MgAl}$ cycle. However, the key feature we observe in NGC 2808 is that the stars appear not to be uniformly distributed along the anticorrelation, but instead it looks like they are clustered in several dis- 


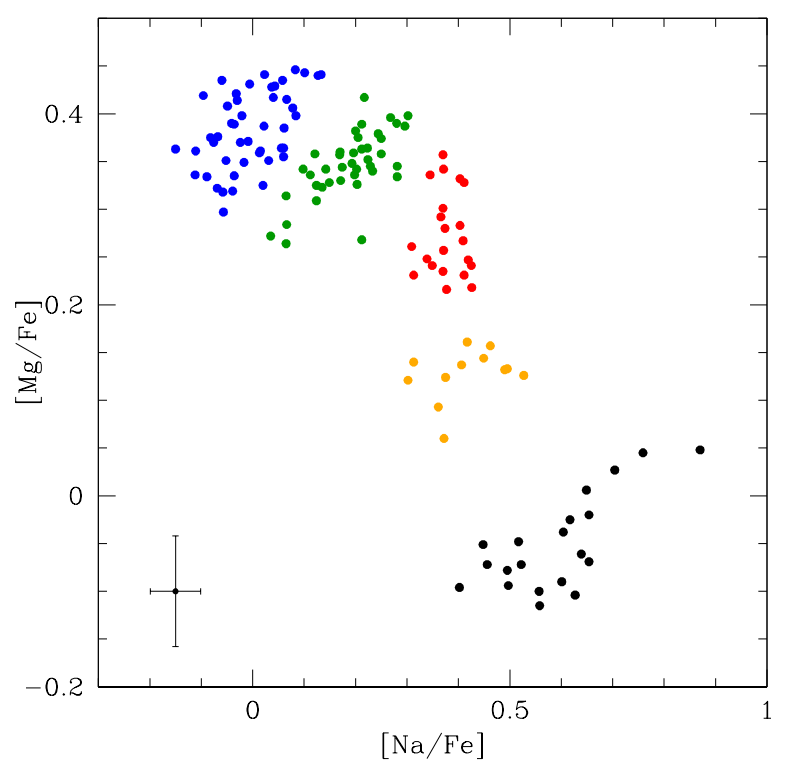

Figure 8. Anticorrelation between abundances of $\mathrm{Mg}$ and $\mathrm{Na}$ in RGB stars of NGC 2808. Different colours are used to indicate giants of different groups. Internal error bars are those derived for GIRAFFE, the ones relative to UVES being slightly smaller.

tinct groups. In analogy with the division based on $\mathrm{O}, \mathrm{Na}$ abundances we call these five groups P1, P2 (with primordial or almost primordial abundance ratios), I1, I2 (with intermediate composition), and E (with severely extreme changes from the original composition).

To check this appearence we examine the distribution function of the $[\mathrm{Na} / \mathrm{Mg}]$ ratio in the left panel of Fig. 9.

The distribution presents four clear peaks, with the group I2 seen as a tail rightward of the I1 groups, because these two groups have quite similar $\mathrm{Na}$ content, but $\mathrm{Mg}$ abundances decreasing by about 0.2 dex from I1 to I2 (see Fig. 8. A clearer representation of the five groups is given by the right panel of Fig. 9 where we plot the distribution function relative to the quantity $[\mathrm{Na} / \mathrm{Fe}]-$ $2[\mathrm{Mg} / \mathrm{Fe}]$ to account for the fact that the spread in $\mathrm{Na}$ is almost twice the spread in $\mathrm{Mg}$. This is a sort of pseudoratio, in analogy with pseudo-colours used in photometry (see e.g. Milone et al. 2015, and references therein).

In this case all five groups are clearly separated, and this plot is a mirror image of the Fig. 7] assessing on a more robust statistical basis the presence of five groups of stars with distinct abundances of proton-capture elements on the RGB of NGC 2808.

We indicate with different colours in Fig 8 these components: blue (P1), green (P2), red (I1), orange (I2), and black (E). Mean abundances for each group are listed in Tab. 17, where Al abundances available for 31 giants with UVES spectra are from Carretta (2014), with atmospheric parameters already on the present homoge-

\footnotetext{
${ }^{4}$ Star 46580 , with $[\mathrm{Na} / \mathrm{Fe}]=+0.212$ dex and $[\mathrm{Mg} / \mathrm{Fe}]=+0.268$, may have a somewhat more uncertain definition, as it is located in an intermediate position between the P2 and I1 groups. Looking at the ratios of the other elements, we assigned this star to the P2 component. Star 55822, with no $\mathrm{Mg}$ determination, has $[\mathrm{O} / \mathrm{Fe}]=$ -0.644 dex, $[\mathrm{Na} / \mathrm{Fe}]=+0.608 \mathrm{dex}$, and it is very likely that this object belongs to the E component. Conservatively, we did not assign this star to any of the five group. None of our results is however affected by these two objects.
}

neous scale.

The fraction of RGB stars in each group is $\mathrm{P} 1=$ $33 \pm 5 \%, \mathrm{P} 2=29 \pm 5 \% \mathrm{I} 1=15 \pm 3 \%, \mathrm{I} 2=9 \pm 2 \%$, and $\mathrm{E}=14 \pm 3 \%$. All groups are well defined and distinct in the $\mathrm{Na}-\mathrm{Mg}$ plane. In Fig. 10 we show how different they are by plotting the cumulative distributions of the $[\mathrm{Na} / \mathrm{Mg}]$ ratio for the five components, using the same colour-coding of Fig. 8

To put on a quantitative foundation these differences, we test with a Kolmogorov-Smirnov test the null hypothesis that any pair of the distributions are extracted from the same parent distribution in $[\mathrm{Na} / \mathrm{Mg}]$. This hypothesis is safely rejected in all cases, with the probabilities of rejecting it by mere chance given in the last column of Tab. 8 .

\section{DISCUSSION}

The homogeneous re-analysis of 140 red giants allows to uncover five different populations, with distinct chemical composition, on the RGB of NGC 2808, thanks to large statistics and a significant decrease of internal errors on abundances. We can look now in more detail to the properties of these components.

\subsection{Chemical tagging of the five groups on the $R G B$}

The separation of RGB stars into five groups has been assessed from both the classical $\mathrm{Na}-\mathrm{O}$ and the $\mathrm{Na}-\mathrm{Mg}$ anticorrelations. The last was used to operatively define the five populations, and with this criterion we plotted with different colour stars on the anticorrelations and the correlation among proton-capture elements $\mathrm{Na}, \mathrm{O}, \mathrm{Mg}$, Si in Fig. 11]

The classification with the $[\mathrm{Na} / \mathrm{Mg}]$ results efficient in ranking the five populations in all the other abundance planes, ordering the P1, P2, I1, I2, and E groups with decreasing $\mathrm{Mg}$, and $\mathrm{O}$ abundances and increasing $\mathrm{Na}$, Si abundances. The boundaries of the populations are however more neatly defined in the Na-Mg plane. Some "blurring" is present at the border between the primordial populations P1 and P2 when $\mathrm{O}$ is involved, although the bulk of stars is well separated. The smearing of stars in $\mathrm{P} 1$ and $\mathrm{P} 2$ appears to be more severe with the Si abundances (bottom-right panel in Fig. 11). However, this is expected because the production of ${ }^{28} \mathrm{Si}$ is not a main process in the proton-capture reactions, but only a leakage from the Mg-Al cycle (Karakas \& Lattanzio 2003). When the bulk of abundances is still primordial, with the large overabundances of $\alpha$-elements from type II supernovae nucleosynthesis still in place, a slight enhancement in $\mathrm{Si}$ from ${ }^{27} \mathrm{Al}(p, \gamma){ }^{28} \mathrm{Si}$ is not clearly discernible. However, as more severe changes in composition from the action of hot H-burning start to appear among stars, also the modification to the Si level becomes more evident, as shown by stars of the I1, I2, and E populations.

Abundances of $\mathrm{Mg}$ and $\mathrm{Al}$ for the 31 giants with UVES spectra were derived in Carretta (2014) using the present homogeneous scale. The Mg-Al anticorrelation shows three clumps of stars with distinct chemical composition and the three discrete populations account for $68 \pm 15 \%, 19 \pm 8 \%$, and $13 \pm 4 \%$ of stars, for the P,I,E components, respectively. A comparison with Carretta (2014) shows the $\mathrm{P}$ stars along the Mg-Al anticorrelation are split among the present $\mathrm{P} 1$ and $\mathrm{P} 2$ groups, the I stars in the I1 and I2, and finally the E stars in Carretta 


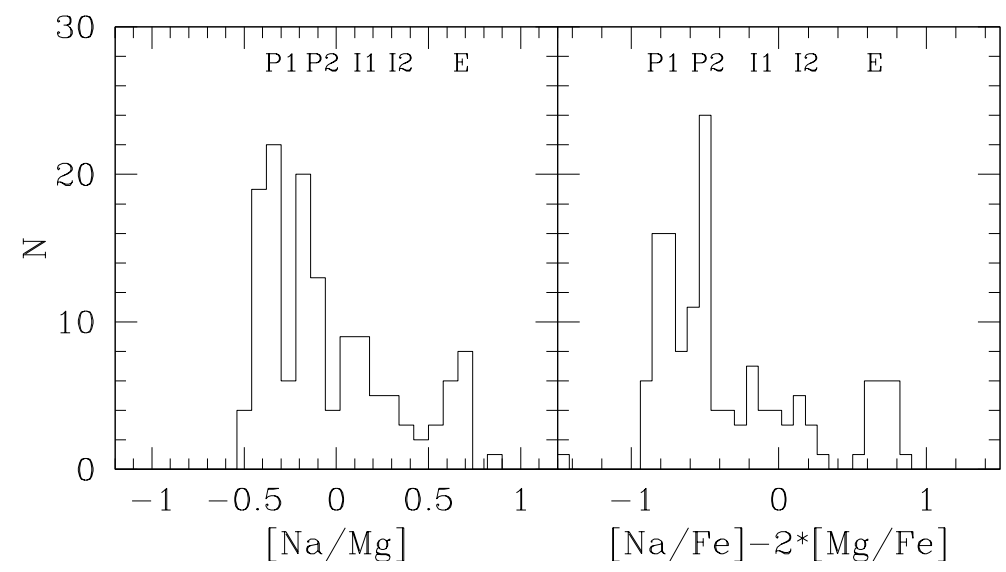

Figure 9. Left panel: distribution function of the $[\mathrm{Na} / \mathrm{Mg}]$ ratio for RGB stars in NGC 2808. In the right panel is plotted the distribution of the quantity $[\mathrm{Na} / \mathrm{Fe}]-2[\mathrm{Mg} / \mathrm{Fe}]$ for the same stars. The five peaks corresponding to the five groups along the Na-Mg anticorrelation are labeled.

Table 7

Average abundances of proton-capture elements in the five groups on the RGB of NGC 2808.

\begin{tabular}{|c|c|c|c|c|c|}
\hline el. & $\mathrm{P} 1$ & $\mathrm{P} 2$ & I1 & $\mathrm{I} 2$ & $\mathrm{E}$ \\
\hline & $\mathrm{n}$ avg. $\mathrm{rms}$ & $\mathrm{n}$ avg. $\mathrm{rms}$ & $\mathrm{n}$ avg. $\mathrm{rms}$ & $\mathrm{n}$ avg. $\mathrm{rms}$ & $\mathrm{n}$ avg. $\mathrm{rms}$ \\
\hline$[\mathrm{Fe} / \mathrm{H}]$ & $46-1.128 \quad 0.028$ & $40-1.1340 .026$ & $21-1.1300 .024$ & $12-1.1370 .028$ & $20-1.1150 .019$ \\
\hline$[\mathrm{O} / \mathrm{Fe}]$ & $42+0.3080 .058$ & $35+0.1540 .090$ & $15-0.2160 .124$ & $7-0.4470 .217$ & $17-0.6560 .161$ \\
\hline$[\mathrm{Na} / \mathrm{Fe}]$ & $46-0.0050 .067$ & $40+0.1880 .068$ & $21+0.3780 .034$ & $12+0.4140 .072$ & $20+0.5920 .112$ \\
\hline$[\mathrm{Mg} / \mathrm{Fe}]$ & $46+0.3840 .041$ & $40+0.3460 .035$ & $21+0.2740 .044$ & $12+0.1270 .028$ & $20-0.0500 .050$ \\
\hline$[\mathrm{Al} / \mathrm{Fe}]$ & $12+0.0650 .062$ & $9+0.2730 .132$ & $4+1.0060 .118$ & $2+1.0790 .108$ & $4+1.2180 .089$ \\
\hline$[\mathrm{Si} / \mathrm{Fe}]$ & $46+0.2650 .026$ & $40+0.2620 .026$ & $21+0.3090 .026$ & $12+0.3460 .038$ & $20+0.3900 .036$ \\
\hline$[\mathrm{Na} / \mathrm{Mg}]$ & $46-0.388 \quad 0.059$ & $40-0.1580 .051$ & $21+0.1040 .057$ & $12+0.2870 .069$ & $20+0.6420 .083$ \\
\hline
\end{tabular}

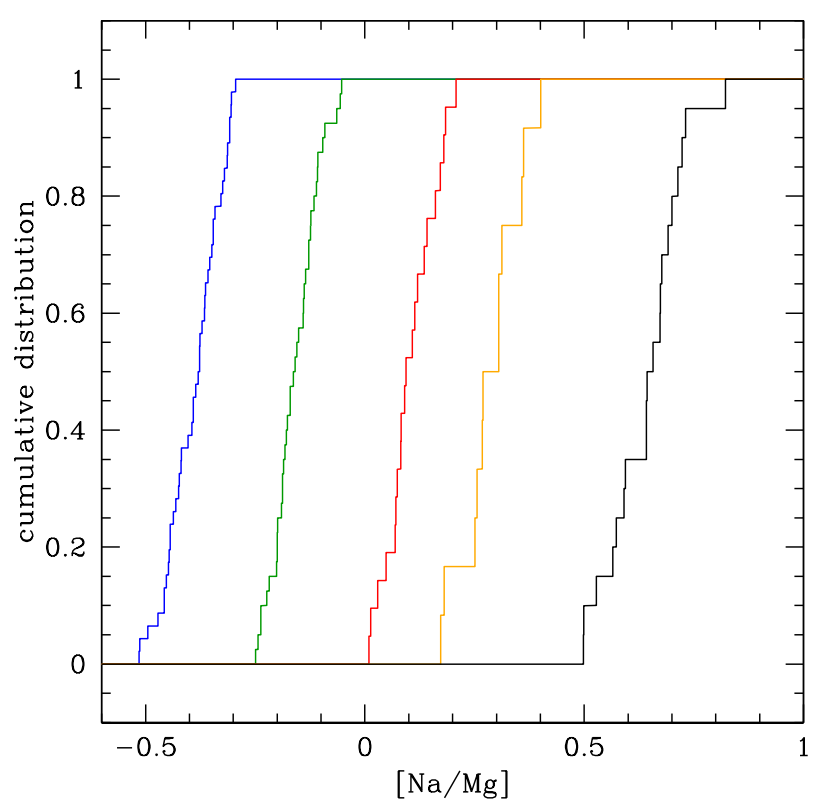

Figure 10. Cumulative distribution of the $[\mathrm{Na} / \mathrm{Mg}]$ ratio in the five groups of RGB stars using the same colour-coding of Fig. 8 blue, green, red, orange, and black indicate groups P1, P2, I1, I2, and $\mathrm{E}$, respectively.

(2014) are found to belong here all to the E population (see Tab. (7) with severe Mg depletions. The fractions derived independently along the $\mathrm{Mg}-\mathrm{Al}$ anticorrelation are in excellent agreement (within the associated Poisson errors) with number counts for the total $\mathrm{P} 1+\mathrm{P} 2(62 \%)$,
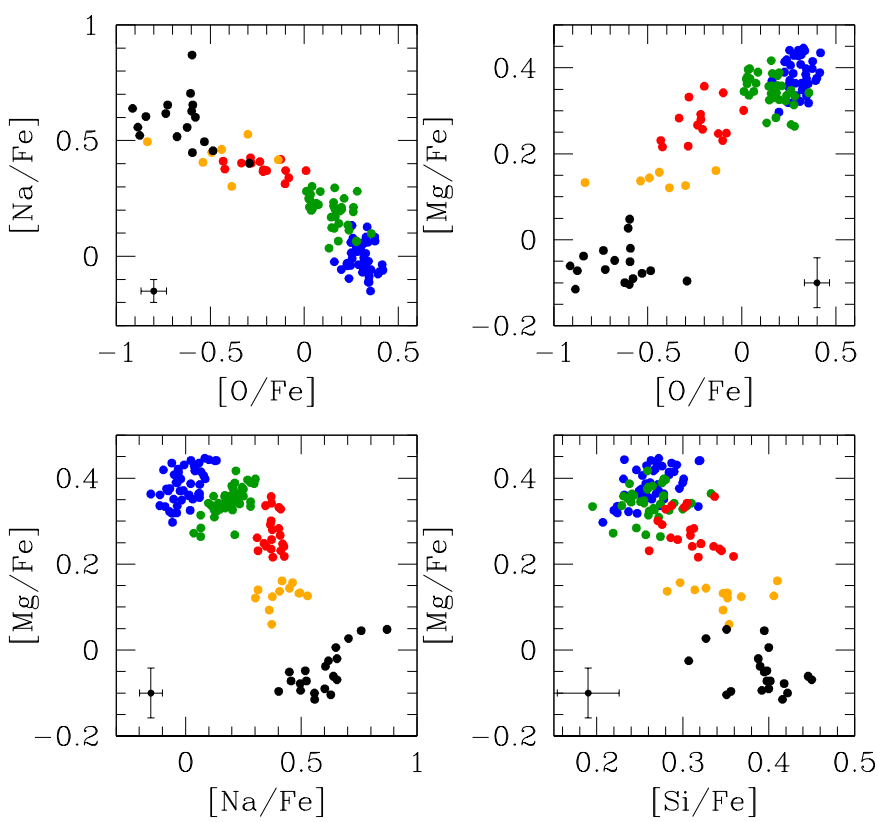

Figure 11. From bottom-left and clockwise: $\mathrm{Mg}-\mathrm{Na}, \mathrm{Na}-\mathrm{O}$ anticorrelations, $\mathrm{Mg}-\mathrm{O}$ correlation and $\mathrm{Mg}-\mathrm{Si}$ anticorrelation among RGB stars in NGC 2808. Different colours indicate the five populations as defined in the $\mathrm{Na}-\mathrm{Mg}$ plane. In each panel the star-to-star error bars are indicated.

I1+I2 (24\%), and E (14\%) populations as defined here from the $\mathrm{Na}-\mathrm{Mg}$ anticorrelation.

A more quantitative description of the chemical com- 
Table 8

t-values, degrees of freedom and two-tailed probability values for the Student t-test on the mean abundance ratios for the five groups in NGC2808. In the last column the probabilities of a K-S test on cumulative distributions of $[\mathrm{Na} / \mathrm{Mg}]$ are also listed.

\begin{tabular}{|c|c|c|c|c|c|c|c|c|}
\hline groups & {$[\mathrm{Fe} / \mathrm{H}]$} & {$[\mathrm{O} / \mathrm{Fe}]$} & {$[\mathrm{Na} / \mathrm{Fe}]$} & {$[\mathrm{Mg} / \mathrm{Fe}]$} & {$[\mathrm{Al} / \mathrm{Fe}]$} & {$[\mathrm{Si} / \mathrm{Fe}]$} & {$[\mathrm{Na} / \mathrm{Mg}]$} & $\operatorname{Prob}(\mathrm{K}-\mathrm{S})$ \\
\hline $\mathrm{P} 1-\mathrm{P} 2$ & 1.03 & 8.73 & 13.22 & 4.64 & 4.38 & 0.53 & 19.39 & $3.5 \times 10^{-20}$ \\
\hline d.f. & 84 & 75 & 84 & 84 & 19 & 84 & 84 & \\
\hline $\mathrm{p}$ & 0.306 & 0.00 & 0.00 & $1.2 \times 10^{-5}$ & $3.2 \times 10^{-4}$ & 0.598 & 0.00 & \\
\hline P1-I1 & 0.30 & 15.76 & 31.00 & 9.69 & 15.26 & 6.43 & 32.41 & $6.0 \times 10^{-14}$ \\
\hline d.f. & 65 & 55 & 65 & 65 & 14 & 65 & 65 & \\
\hline $\mathrm{p}$ & 0.765 & 0.00 & 0.00 & 0.00 & 0.00 & $2.0 \times 10^{-8}$ & 0.00 & \\
\hline P1-I2 & 0.99 & 9.15 & 18.21 & 25.46 & 12.93 & 6.97 & 31.06 & $1.5 \times 10^{-9}$ \\
\hline d.f. & 56 & 47 & 56 & 56 & 12 & 56 & 56 & \\
\hline $\mathrm{p}$ & 0.326 & 0.00 & 0.00 & 0.00 & $2.0 \times 10^{-8}$ & $1.5 \times 10^{-5}$ & 0.00 & \\
\hline P1-E & 2.19 & 24.06 & 22.18 & 34.15 & 24.04 & 14.02 & 50.25 & $1.6 \times 10^{-13}$ \\
\hline d.f. & 64 & 57 & 64 & 64 & 14 & 64 & 64 & \\
\hline $\mathrm{p}$ & 0.032 & 0.00 & 0.00 & 0.00 & 0.00 & 0.00 & 0.00 & \\
\hline P2-I1 & 0.60 & 10.44 & 14.54 & 6.50 & 9.96 & 6.71 & 17.67 & $32.3 \times 10^{-13}$ \\
\hline d.f. & 59 & 48 & 59 & 59 & 11 & 59 & 59 & \\
\hline $\mathrm{p}$ & 0.551 & 0.00 & 0.00 & $2.0 \times 10^{-8}$ & $7.7 \times 10^{-7}$ & 0.00 & 0.00 & \\
\hline P2-I2 & 0.33 & 7.20 & 9.66 & 22.36 & 9.14 & 7.17 & 20.71 & $2.7 \times 10^{-9}$ \\
\hline d.f. & 50 & 40 & 50 & 50 & 9 & 50 & 50 & \\
\hline $\mathrm{p}$ & 0.743 & $1.0 \times 10^{-8}$ & 0.00 & 0.00 & $7.5 \times 10^{-6}$ & 0.00 & 0.00 & \\
\hline P2-E & 3.21 & 19.33 & 14.82 & 31.74 & 15.10 & 14.16 & 39.53 & $5.6 \times 10^{-13}$ \\
\hline d.f. & 58 & 50 & 58 & 58 & 11 & 58 & 58 & \\
\hline $\mathrm{p}$ & 0.002 & 0.00 & 0.00 & 0.00 & $1.0 \times 10^{-8}$ & 0.00 & 0.00 & \\
\hline I1-I2 & 0.73 & 2.62 & 1.63 & 11.71 & 0.76 & 3.00 & 7.79 & $7.0 \times 10^{-6}$ \\
\hline d.f. & 31 & 20 & 31 & 31 & 4 & 31 & 31 & \\
\hline $\mathrm{p}$ & 0.471 & 0.016 & 0.113 & 0.00 & 0.490 & 0.005 & $1.0 \times 10^{-8}$ & \\
\hline I1-E & 2.22 & 8.71 & 8.19 & 21.98 & 2.87 & 8.22 & 24.08 & $3.3 \times 10^{-10}$ \\
\hline d.f. & 39 & 30 & 39 & 39 & 6 & 39 & 39 & \\
\hline $\mathrm{p}$ & 0.032 & 0.00 & 0.00 & 0.00 & 0.028 & 0.00 & 0.00 & \\
\hline $\mathrm{I} 2-\mathrm{E}$ & 2.41 & 2.30 & 7.96 & 12.83 & 1.57 & 3.23 & 13.04 & $1.0 \times 10^{-7}$ \\
\hline d.f. & 30 & 22 & 30 & 30 & 4 & 30 & 30 & \\
\hline $\mathrm{p}$ & 0.022 & 0.031 & $1.0 \times 10^{-8}$ & 0.00 & 0.192 & 0.003 & 0.00 & \\
\hline
\end{tabular}

position in the five populations od RGB stars is summarised in Tab. 8. We used Student's and Welch's tests to determine if two sets of data are significantly different from each other, for every one of the 10 combinations of the five groups. We tested the null hypothesis that any pair of components are extracted from a distribution having the same mean [element/Fe], for $[\mathrm{Fe} / \mathrm{H}],[\mathrm{O} / \mathrm{Fe}]$, $[\mathrm{Na} / \mathrm{Fe}],[\mathrm{Mg} / \mathrm{Fe}],[\mathrm{Al} / \mathrm{Fe}],[\mathrm{Si} / \mathrm{Fe}]$, and $[\mathrm{Na} / \mathrm{Mg}]$. For any combination and element we list the t-value, the number of degrees of freedom, and the two-tailed probability.

The first consideration is that the metallicity $[\mathrm{Fe} / \mathrm{H}]$ is the same for almost all populations. Only the value of the E population seems to be statistically different from the metallicity of the primordial and intermediate components. This result is in qualitative agreement with the findings of Bragaglia et al. (2010) in a large set of GCs and in NGC 2808 in particular, and with the expectation that the extreme population E must be enhanced in helium. By consequence for a fixed global metallicity a decrease in the $\mathrm{H}$ abundance is predicted, so that the ratio $[\mathrm{Fe} / \mathrm{H}]$ is expected to slightly increase.

Mean $\mathrm{O}$ abundances differ among all groups and despite a couple of mutual interlopers (Fig. 11] top left panel) the average abundances of populations P1 and P2 are statistically different. For $\mathrm{Na}$, average abundances do not significantly differ only among the two intermediate populations I1 and I2. The reality of this feature is also confirmed by the fact that their mean abundances of $\mathrm{Al}$ (that correlates with $\mathrm{Na}$ ) are also not significantly different. As already noted by visual inspection of Fig. 11. the mean $[\mathrm{Si} / \mathrm{Fe}]$ ratio is similar for the primordial groups P1 and P2, but it differ in any other case. Finally, the $[\mathrm{Mg} / \mathrm{Fe}]$ and $[\mathrm{Na} / \mathrm{Mg}]$ ratios are found to be statistically different in all populations at a very high level of confidence.

Each of the five populations selected from the $\mathrm{Na}-\mathrm{Mg}$ plane seems to be a single stellar population. Following the approach used by Gratton et al. (2011) for RHB stars, typical (anti)correlations would be observable if a single group were composed by more than one population with homogeneous composition. We verified this issue by looking at the presence of a $\mathrm{Mg}-\mathrm{Na}$ anticorrelation or a $\mathrm{Mg}-\mathrm{O}$ correlation within each group. In each case we found either the reverse relation $(\mathrm{Mg}$ correlated with $\mathrm{Na}$ or anti-correlated with $\mathrm{O}$ ) or the correct relation was found not to be statistically robust. Moreover, in each population the $[\mathrm{Na} / \mathrm{Mg}]$ ratio presents a single peaked distribution.

Recently Mucciarelli et al. (2015) (Mu15) derived potassium abundances for 116 giants of our sample in NGC 2808 (excluding three stars flagged as possible binaries). They found that this GC is the second one after NGC 2419 Mucciarelli et al. 2012; Cohen and Kirby 2012) where a Mg-K anticorrelation is clearly observed. In Fig. 12 we show our $[\mathrm{Mg} / \mathrm{Fe}]$ ratios as a function of their $[\mathrm{K} / \mathrm{Fe}]$ values, putting the $\mathrm{Mg}-\mathrm{K}$ anticorrelation on a larger statistical basis and confirming that $K$ in a few GCs participates to the same self-enrichment network due to proton-capture reactions. A more precise comparison with the results of Mucciarelli et al. (2015) 


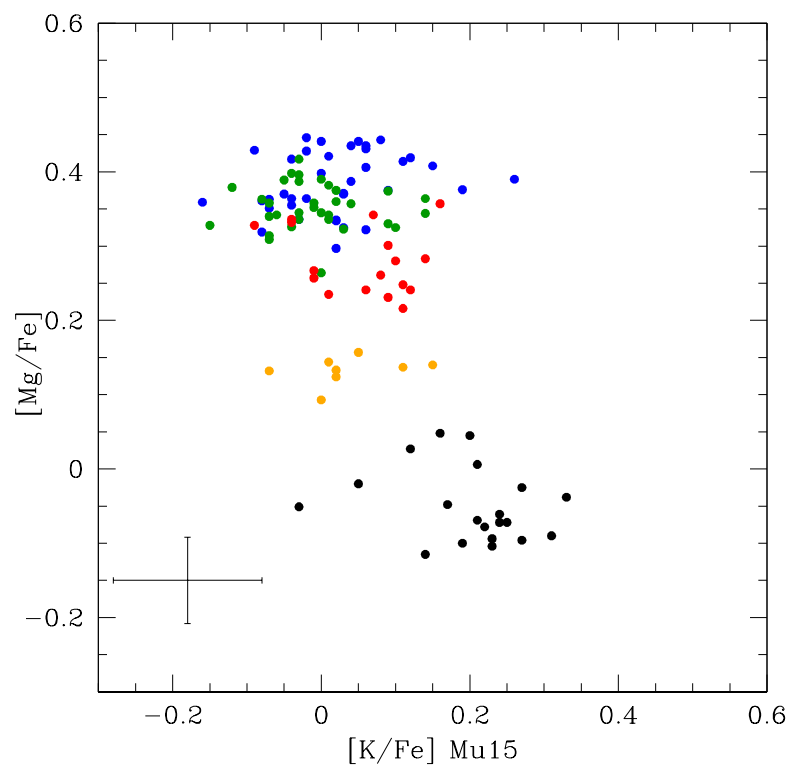

Figure 12. The ratio $[\mathrm{Mg} / \mathrm{Fe}]$ as a function of the $[\mathrm{K} / \mathrm{Fe}]$ ratio from Mucciarelli et al. (2015) in giants of NGC 2808, using the same colour-coding of Fig. 8 for stars of populations P1, P2, I1, I2, and E.

is hampered by the fact that they used for NGC 2808 the older temperature scale by Carretta et al. (2006, 2009a). However, there is a way to bypass this obstacle.

The $\mathrm{Si}-\mathrm{Al}$ correlation and the mirroring $\mathrm{Mg}-\mathrm{Si}$ anticorrelation, observed in NGC 2808 and other GCs (Yong et al. 2005; Carretta et al. 2009a), are thermometers indicating that the temperature of the $\mathrm{H}$ burning generating this pattern exceeded $\mathrm{T}_{6} \sim 65$ $\mathrm{K}$ (Arnould et al. 1999), because at this temperature the reaction ${ }^{27} \mathrm{Al}(p, \gamma){ }^{28} \mathrm{Si}$ becomes dominant over ${ }^{27} \mathrm{Al}(p, \alpha)^{24} \mathrm{Mg}$. However, this is only a lower limit to the temperature, meaning that from now on the leakage on ${ }^{28} \mathrm{Si}$ from the $\mathrm{Mg}$ - $\mathrm{Al}$ cycle is possible. A more elevated temperature regime is required to explain the changes in the K content as observed in NGC 2419 (Ventura et al. 2012). In their attempt to account for the extreme K-enhanced, Mg-depleted stars observed by Mucciarelli et al. (2012), Ventura et al. postulated that at temperatures above $10^{8} \mathrm{k}$ is activated a process of proton-capture on ${ }^{36} \mathrm{Ar}$ that produces potassium and shifts the equilibrium among various species toward heavier nuclei like $\mathrm{K}, \mathrm{Ca}$, and $\mathrm{Sc}$, in particular when low metallicity models are considered. The effect is relevant on K (see Fig. 12) and on Sc, because they are much less abundant that Ca: a fraction of the original Ar is sufficient to provide large abundance variation. Different is the case of $\mathrm{Ca}$, since the original content of this element is larger than that of $\mathrm{K}$ and much more abundant than Sc.

Hence, if this process were at work also in (at least part of) first generation stars at the higher metallicity of NGC 2808, we could test it by using our homogeneous set of abundances without being afraid of possible offests between different analyses, as for K, since with the same scale we derived in the present work abundances of Sc, $\mathrm{Mg}, \mathrm{Si}$, and $\mathrm{Ca}$. The relations between these elements are
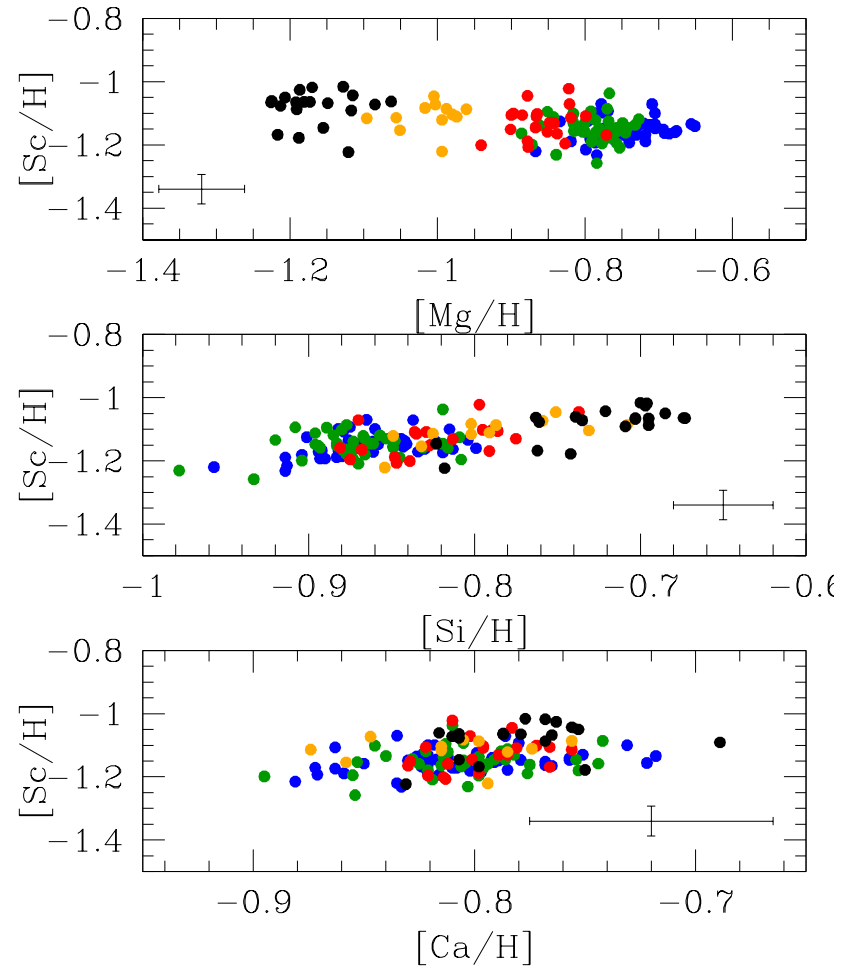

Figure 13. From top to bottom, Sc as a function of the abundances of $\mathrm{Mg}, \mathrm{Si}$, and $\mathrm{Ca}$ in our sample. Internal error bars are shown in each panel.

shown in Fig.13. The probability that $\mathrm{Sc}$ and $\mathrm{Mg}$ are not anticorrelated is $\mathrm{P}=1.0 \times 10^{-8}$ whereas that of $\mathrm{Sc}$ and $\mathrm{Si}$ lacking a correlation is $\mathrm{P}=0.0$. For the correlation $\mathrm{Sc}-\mathrm{Ca}$ we found $\mathrm{P}=3.5 \times 10^{-5}$, with 140 d.o.f. Finally, the correlations of Sc and Si with K are both found to be statistically significant. We may conclude that clear evidence does exist for the action of proton-capture occurring at a particularly high temperature regime in a part of stars that contributed to the pollution of intracluster gas at the epoch of the formation of the following generation(s) of stars. A clue on the origin of this processed material is however hard to assess since such temperatures are encountered in the two main candidate polluters proposed for GCs, namely massive AGBs and the most massive main-sequence stars (see Prantzos et al. 2007).

The cases of NGC 2419 and NGC 2808 point out, albeit at a different degree, the key rôle of the Mg-depletion as useful indicator of a particular environment where the network of proton-capture reactions operate at unusually high temperature. We may then confirm the plot in Fig. 14 first introduced in Carretta et al. (2013b), as a powerful diagnostic to uncover GCs where this extreme processing occurred. In this plot we compare one of the element most affected by this advanced nucleosynthesis, $\mathrm{Mg}$, with one of the least varied, Ca. Using as a reference the homogeneous analysis of more than 280 RGB stars with UVES spectra in 23 GCs (green squares) it is possible to pick up clusters were signatures of such a particular nucleosynthesis are visible. Apart from the exceptional case of NGC 2419, only NGC 2808, NGC 4833 and M 15 stand out, with the last two GCs being much more metal-poor. 


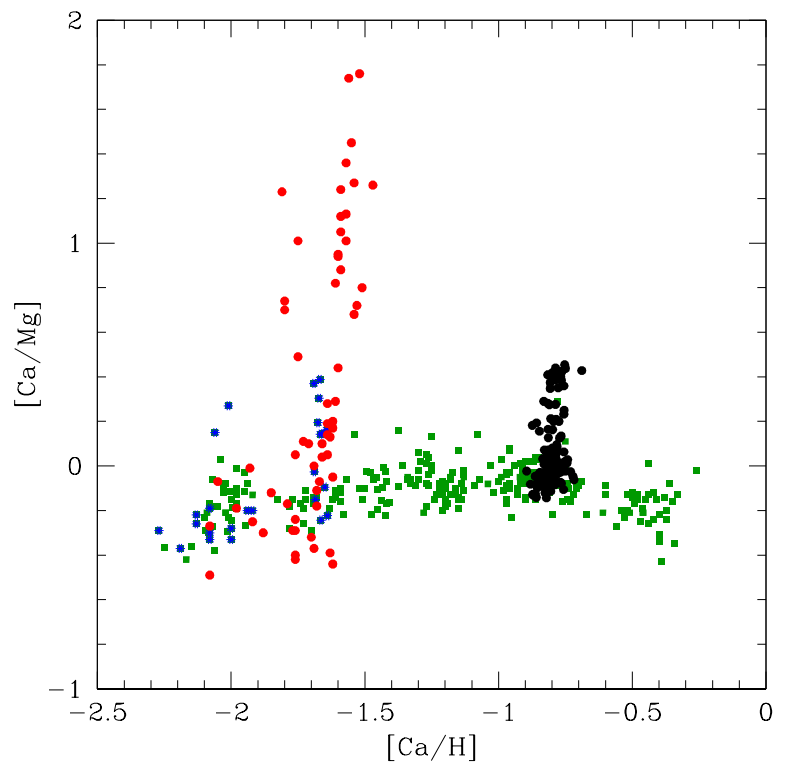

Figure 14. The ratio $[\mathrm{Ca} / \mathrm{Mg}]$ as a function of the $[\mathrm{Ca} / \mathrm{H}]$ ratio for giants in NGC 2419 (Mucciarelli et al. 2012; Cohen and Kirby 2012, red filled circles), in about 280 RGB stars in more than 20 GCs (Carretta et al. 2009a, 2010b, 2011, 2013a, 2014, 2015, 2010d, green squares), and giants in NGC 2808 (present work; black circles). Blue asterisks indicate M 15 and NGC 4833.

\subsection{Comparison with photometry}

Milone et al. (2015) identified at least five clumps of stars on the RGB in NGC 2808 from precise UV photometry with $H S T$ and colour or pseudo-colour indexes purposedly tailored to maximize the separation among photometric sequences due to the absorption of molecular bands like $\mathrm{OH}, \mathrm{NH}, \mathrm{CN}$, and $\mathrm{CH}$ of elements involved in proton-capture reactions. The counterparts of these discrete populations are also found among MS stars, although less clearly since molecular bands become less prominent in warmer stars. Using 32 giants in their set with abundances from Carretta et al. (2004, 2006) and Carretta (2014) Milone et al. showed that their populations from B to E present signatures of increasingly processed composition, namely depletions in $\mathrm{O}$ and $\mathrm{Mg}$ and enhancements in $\mathrm{Na}$ and $\mathrm{Al}$. The metallicity and composition of their population A is unknown, lacking stars with spectroscopy, but they inferred a primordial abundance pattern in this group.

A direct comparison is difficult because the photometric catalogue are not yet published and usually $H S T$ fields are taken on the cluster centre, with scarce overlap with the pointings of multiobject spectrographs like FLAMES. However, we can use the 32 stars in common to bridge the gap and compare the five groups detected photometrically to the five populations with distinct composition uncovered in the present work. For each group of stars associated by Milone et al. (2015) to populations $\mathrm{B}, \mathrm{C}, \mathrm{D}, \mathrm{E}$ we recomputed the average and $r m s$ scatter of the $[\mathrm{O} / \mathrm{Fe}],[\mathrm{Na} / \mathrm{Fe}],[\mathrm{Mg} / \mathrm{Fe}]$, and $[\mathrm{Si} / \mathrm{Fe}]$ ratios using the presently derived new abundances. Al was not considered, since only a few stars from Carretta (2014) are in the photometric sample (all in the primordial regime, three in group B and two in C). We used these values simply to locate the position of the photometric groups in the upper half of the four panels in
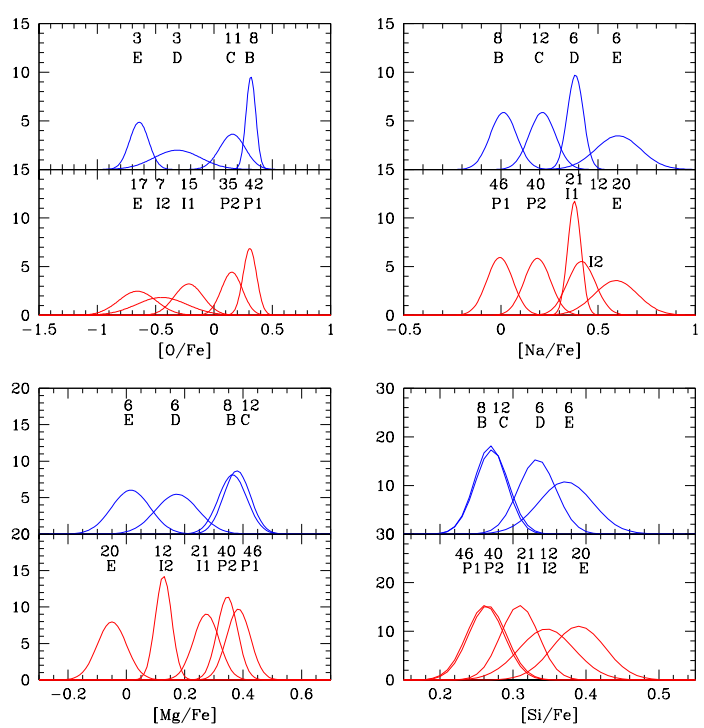

Figure 15. Comparison of mean abundance ratios for the photometric populations B,C,D,E identified by Milone et al. (2015), upper half of each panel)) with average ratios of the five populations $\mathrm{P} 1, \mathrm{P} 2, \mathrm{I} 1, \mathrm{I} 2, \mathrm{E}$ (lower half of each panel). Each population is represented by a gaussian centered at the mean value and with $\sigma$ given by the rms scatter of the mean ratio.

Fig. 15, representing each group as a gaussian centered at the elemental mean ratio, and having the corresponding rms scatter as $\sigma$.

In the bottom half of each panel we plotted the position of our five populations using the values in Tab. 7. This comparison shows that the match is satisfactory but not perfect. The cross-identification of a few populations, namely the extreme components, seems to be firmly established. The two groups P1 and P2 with primordial or almost primordial composition correspond to the populations B and C, respectively, for each of the four elements. We may conclude that the parent populations of the two groups of RHB stars in NGC 2808 are then traced on the RGB using both photometry and spectroscopy. Also the extreme population is easily (and unambigously) identified in the present work and in the $H S T$ photometry by Milone and collaborators.

Some problems seem to arise when dealing with the intermediate components. In the panels of Fig. 15 for $\mathrm{O}, \mathrm{Mg}$, and $\mathrm{Si}$ the photometric component $\mathrm{D}$ looks more or less coincident with our population I2: in these plots seems difficult to trace among the photometric groups the counterpart of I1, the other population with intermediate composition. On the other hand, when looking at $\mathrm{Na}$, the photometric group D seems to be associated more to the I1 population than to I2. However, this mismatch may actually be only apparent, because in Fig. 3 of Milone et al. (2015) group D shows a large spread in the index $\Delta_{\mathrm{F} 336 \mathrm{~W}}$.F438W, with a tail visible at low values. Since this index is sensitive to the abundances of light elements $(\mathrm{C}, \mathrm{N})$, it is likely that also the group $\mathrm{D}$ is not a simple stellar population, as already stated for groups $\mathrm{B}$ and $\mathrm{C}$ by Milone et al.

In Tab. 9 we summarized recent results from spectroscopy and photometry to estimate the number of components detected in different evolutionary phases in 
Table 9

Stellar populations in NGC 2808

\begin{tabular}{|c|c|c|c|c|}
\hline $\begin{array}{l}\text { Na-O RGB } \\
\text { fraction }\end{array}$ & $\begin{array}{c}\mathrm{P} \\
45 \pm 6 \%\end{array}$ & $\begin{array}{c}\mathrm{I} \\
36 \pm 6 \%\end{array}$ & $\begin{array}{c}\mathrm{E} \\
18 \pm 4 \%\end{array}$ & this work \\
\hline $\begin{array}{l}\mathrm{Na}-\mathrm{Mg} \mathrm{RGB} \\
\text { fraction }\end{array}$ & $\begin{array}{cc}\mathrm{P} 1 & \mathrm{P} 2 \\
33 \pm 5 \% & 29 \pm 5 \%\end{array}$ & $\begin{array}{cc}\mathrm{I} 1 & \mathrm{I} 2 \\
15 \pm 3 \% & 9 \pm 2 \%\end{array}$ & $\begin{array}{c}\mathrm{E} \\
14 \pm 3 \%\end{array}$ & this work \\
\hline $\begin{array}{l}\mathrm{Mg}-\mathrm{Al} \mathrm{RGB} \\
\text { fraction }\end{array}$ & $\begin{array}{c}\mathrm{P} \\
68 \pm 15 \%\end{array}$ & $\begin{array}{c}\mathrm{I} \\
19 \pm 8 \%\end{array}$ & $\begin{array}{c}\mathrm{E} \\
13 \pm 4 \%\end{array}$ & Carretta 2014 \\
\hline $\begin{array}{l}\text { from O RGB } \\
\text { fraction }\end{array}$ & $\begin{array}{l}\text { O-normal } \\
61 \pm 17 \%\end{array}$ & $\begin{array}{l}\text { O-poor } \\
22 \pm 4 \%\end{array}$ & $\begin{array}{c}\text { super O-poor } \\
17 \pm 4 \%\end{array}$ & Carretta+ 2006 \\
\hline Na-O HB & $\begin{array}{c}\text { RHB } \\
\text { O-rich/Na-poor } \\
\text { moderate Na-O } \\
\text { anticorrelation }\end{array}$ & $\begin{array}{c}\text { BHB } \\
\text { O-poor/Na-rich }\end{array}$ & & Gratton+ 2011 \\
\hline $\mathrm{Na}-\mathrm{O} \mathrm{HB}$ & $\begin{array}{c}\text { RHB } \\
\text { lower } \mathrm{Na} \\
\text { bimodal } \mathrm{Na}\end{array}$ & $\begin{array}{l}\text { BHB } \\
\text { higher } \mathrm{Na} \\
\text { higher } \mathrm{He}\end{array}$ & & Marino+ 2014 \\
\hline $\mathrm{K}$ in $\mathrm{RGB}$ & $\begin{array}{l}\text { Primordial } \\
\text { low K }\end{array}$ & $\begin{array}{c}\text { Intermediate } \\
\text { intermediate K }\end{array}$ & $\begin{array}{l}\text { Extreme } \\
\text { high K }\end{array}$ & Mucciarelli+ 2015 \\
\hline \multicolumn{5}{|c|}{ photometry } \\
\hline $\begin{array}{l}\text { MS } \\
\text { fraction }\end{array}$ & $\begin{array}{c}\text { rMS } \\
62 \pm 2 \%\end{array}$ & $\begin{array}{c}\mathrm{mMS} \\
24 \pm 2 \%\end{array}$ & $\begin{array}{c}\text { bMS } \\
14 \pm 3 \%\end{array}$ & Milone+ 2012 \\
\hline $\begin{array}{l}\text { AGB } \\
\text { fraction }\end{array}$ & $\begin{array}{c}\operatorname{AGB}(\mathrm{I}) \\
49 \pm 11 \%\end{array}$ & $\begin{array}{l}\mathrm{AGB}(\mathrm{II}) \\
22 \pm 6 \%\end{array}$ & $\begin{array}{l}\mathrm{AGB}(\mathrm{III}) \\
29 \pm 8 \%\end{array}$ & Milone+ 2015 \\
\hline $\begin{array}{l}\text { AGB } \\
\text { fraction }\end{array}$ & $\begin{array}{c}\text { A B C } \\
\text { A } 5.8 \pm 0.5 \% \\
\text { B } 17.4 \pm 0.9 \% \\
\text { C } 26.4 \pm 1.2 \%\end{array}$ & $\begin{array}{c}\mathrm{D} \\
31.3 \pm 1.3 \%\end{array}$ & $\begin{array}{c}\mathrm{E} \\
19.1 \pm 1.0 \%\end{array}$ & Milone+ 2015 \\
\hline $\begin{array}{l}\text { estimate } \\
\text { from MS } \\
\text { from RGB }\end{array}$ & $\begin{array}{ccc}\text { A B C } & \\
\mathrm{Y}=0.246 & 0.278 & 0.280 \\
\mathrm{Y}=0.243 & 0.278 & 0.280\end{array}$ & $\begin{aligned} & \mathrm{D} \\
\mathrm{Y} & =0.329 \\
\mathrm{Y} & =0.318\end{aligned}$ & $\begin{array}{c}E \\
Y=0.384 \\
Y=0.367\end{array}$ & Milone+2015 \\
\hline $\begin{array}{l}\text { from } H B \\
\text { fraction } \\
\text { estimate }\end{array}$ & $\begin{array}{c}\text { RHB } \\
41 \pm 3 \% \\
\mathrm{Y}=0.248\end{array}$ & $\begin{array}{c}\text { BHB } \\
39 \pm 3 \% \\
\mathrm{Y}=0.30\end{array}$ & $\begin{array}{c}\text { EHB } \\
9 \pm 1 \% \\
Y=0.40\end{array}$ & Dalessandro+ 2011 \\
\hline $\begin{array}{l}\text { from HB } \\
\text { fraction }\end{array}$ & $\begin{array}{c}\text { RHB } \\
41 \pm 3 \%\end{array}$ & $\begin{array}{c}\text { EBT1 } \\
34 \pm 2 \%\end{array}$ & $\begin{array}{c}\text { EBT2 } \\
14 \pm 1 \%\end{array}$ & Iannicola +2009 \\
\hline $\begin{array}{l}\text { from } \mathrm{HB} \\
\text { fraction } \\
\text { estimate }\end{array}$ & $\begin{array}{c}\text { RHB } \\
50 \% \\
Y=0.24\end{array}$ & $\begin{array}{c}\text { EBT1 } \\
30 \% \\
\mathrm{Y}=0.26-0.29\end{array}$ & $\begin{array}{c}\text { EBT2 } \\
20 \% \\
Y=0.40\end{array}$ & D'Antona + 2005 \\
\hline $\begin{array}{l}\text { from HB } \\
\text { fraction }\end{array}$ & $\begin{array}{c}\text { RHB } \\
46 \pm 10 \% \\
\end{array}$ & $\begin{array}{c}\text { EBT1 } \\
35 \pm 10 \% \\
\end{array}$ & $\begin{array}{c}\text { EBT2 } \\
10 \pm 5 \% \\
\end{array}$ & Bedin +2000 \\
\hline
\end{tabular}

Notes: residual components on the HB are as follows:

EBT3 $9 \pm 5 \%($ Bedin +2000$)$

BHk $9 \pm 1 \%$ (blue hook stars, Dalessandro+2011)

EBT3 $11 \pm 1 \%$ (Iannicola +2009$)$ 


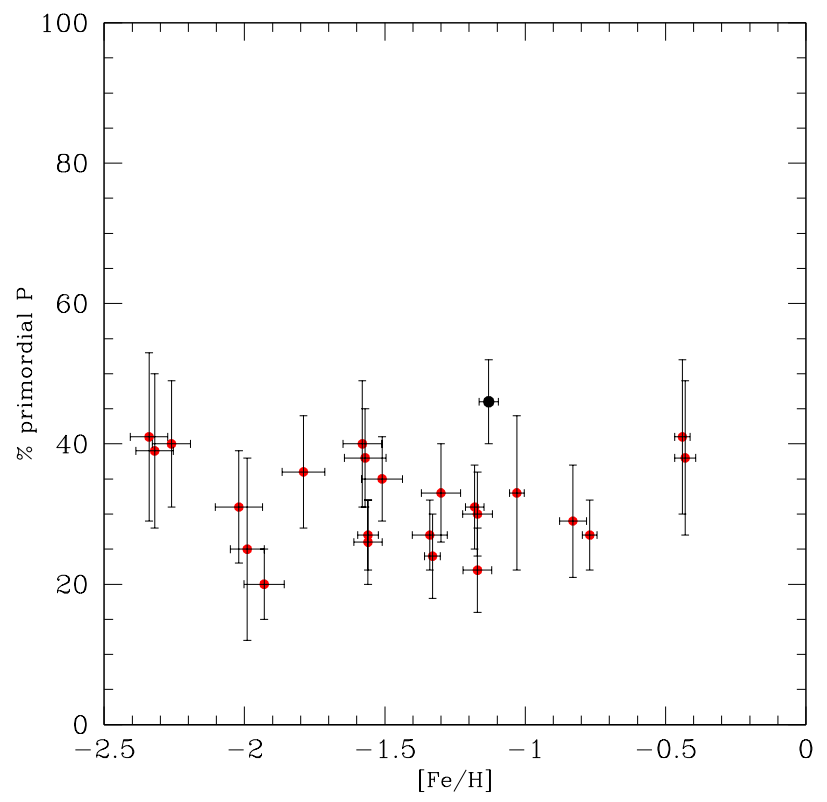

Figure 16. Fractions of first generation stars in 24 GCs of our FLAMES survey as a function of the metallicity $[\mathrm{Fe} / \mathrm{H}]$. Attached error bars in fractions are from Poisson's statistics. For metallicity we used systematic errors (from Tab. 3 and similar tables in the other papers). NGC 2808 is indicated with a larger black symbol.

NGC 2808, and the fractions of stars attributed to each one. Despite the variety of methods, and evolutionary phases sampled a few facts emerge.

First, NGC 2808 hosts the largest fraction of stars with primordial composition observed among massive Milky Way GCs up to date: about 50\% of the currently observed stellar population belongs to the original generation formed in this object. This feature is clearly appreciated in Fig. 16, based on the now fully homogeneous analysis of $\mathrm{Na}, \mathrm{O}$ abundances for 1598 red giants in 24 GCs from our FLAMES survey, where we plot the fraction of stars in the $\mathrm{P}$ component as a function of metallicity. The estimates for NGC 2808 range from about $41 \%$ from number count on the RHB (Iannicola et al. 2009; Dalessandro et al. 2011) up to the maximum value of $68 \%$ derived from the $\mathrm{Mg}-\mathrm{Al}$ anticorrelation in RGB stars (Carretta 2014). Even such a high value seems however confirmed by number counts on the red MS (Milone et al. 2012b). Since on average the stellar primordial content in GCs is much lower (about 30\% as estimated from the $\mathrm{Na}-\mathrm{O}$ anticorrelation) an open question is how and why NGC 2808 retained such a higher fraction.

The second feature inferred from Tab. 9 is that a gross division into three components seems to be accounted for by most analysis. For the intermediate group, with clearly modified but not extreme chemical composition, a fraction ranging from one fifth up to one third is observed by most studies. The extreme component is also traced (whenever accessible to observations) by most works, with reassuringly similar estimates of the fraction of stars.

However, a third fact is assessed by recent studies, although hints were already suggested by early investigations of the multimodal HB in NGC 2808: a finer subdivision of components is corroborated from high precision photometry and spectroscopy. The cross match between abundances and colour indexes is usually good and mutually suggests that what we are observing are real stellar populations forming discrete sequences or groups.

\section{SUMMARY AND CONCLUSIONS}

From high/intermediate spectra we reanalysed 140 individual RGB stars in NGC 2808 using the same homogeneous procedures adopted for more than 2500 giants in other $23 \mathrm{GCs}$. We derived abundances of Fe, O, $\mathrm{Na}, \mathrm{Mg}, \mathrm{Si}, \mathrm{Ca}, \mathrm{Sc}, \mathrm{Ti}, \mathrm{Cr}, \mathrm{Mn}, \mathrm{Ni}$. Pure $\alpha$-elements and elements of the Fe-group are constant among multiple populations in NGC 2808. On our high resolution UVES metallicity scale (Carretta et al. 2009c) we found a metal abundance for NGC 2808 of $[\mathrm{Fe} / \mathrm{H}]=$ $-1.129 \pm 0.005 \pm 0.034$ ( \pm statistical \pm systematic error) with $\sigma=0.030$ (31 stars).

We confirm that the Na-O anticorrelation in this cluster is one of the most extended observed in GCs with no intrinsic spread in iron, in agreement with the high cluster present day total mass and with the hot temperatures reached on the blue HB. According to the definition of Carretta et al. (2009b) NGC 2808 host the highest fraction of the primordial, first generation stars detected spectroscopically. The improved statistics and the smaller internal errors due to uncertainties in the analysis uncover that stars along the $\mathrm{Na}-\mathrm{O}$ anticorrelation seems clustered in five groups.

The larger sample of stars with $\mathrm{Na}, \mathrm{Mg}$ abundances allow to confirm that five populations of RGB stars with distinct composition of proton-capture elements are located along the Na-Mg anticorrelation. Statistical tests provide robust evidence of the different chemistry of these components that we called P1, P2, I1, I2, and E in order of decreasing $\mathrm{Mg}$ and increasing $\mathrm{Na}$ abundances. Large Mg depletions are observed in NGC 2808, with a fraction of about $14 \%$ of stars (the $\mathrm{E}$ component) having solar or even subsolar $[\mathrm{Mg} / \mathrm{Fe}]$ ratios.

We confirm and extend in statistics the anticorrelation between $\mathrm{Mg}$ and potassium abundances found by Mucciarelli et al. (2015) in this GC. Mg anticorrelated with $\mathrm{K}$ and $\mathrm{Si}$, as well as with $\mathrm{Sc}$, is proof that protoncapture processes operated in a very elevated temperature regime for $\mathrm{H}$-burning in part of stars of the first generation in NGC 2808.

The match of the five populations detected from spectroscopy and the five groups recently discovered by Milone et al. (2015) with UV photometry is good but not perfect. The two populations with almost primordial abundances are well cross-identified, nicely tracing the progenitors of the two groups of RHB stars individuated by Gratton et al. (2011) and Marino et al. (2014). There is also a definite agreement on the extreme component. The partial mismatch of the populations with intermediate composition confirm the evidence that other discrete components may exist in NGC 2808, as suggested by the photometry.

Apparently, current modelling seems to lag somewhat behind observations. The strong depletions detected in NGC 2808 (Carretta 2014, and the present analysis) offer a severe challenge to the main scenario of selfenrichment of GCs in early epochs. Models of asymptotic giant branch (AGB) or SAGB stars with the proper metallicity $(\mathrm{Z}=0.001)$ produce the required depletions 
in $\mathrm{Mg}$ (Ventura et al. 2013), but not enough to match the reduction factors observed in NGC 2808. On the other hand, the wind from model $60 \mathrm{rD}$ in the set of fast rotating massive stars (FRMS) by Decressin et al. (2007) seems to be able to account for a large range of $\mathrm{Mg}$ depletions and $\mathrm{Al}$ enhancement, but at the price of a strong destruction of $\mathrm{Na}$, which is not observed in stars of NGC 2808 with extreme composition (this work and Carretta 2014). For the time being, we note that Bastian et al. (2015) showed how no one of the most fashionable models proposed for polluting first generation stars is able to fit at the same time the abundance pattern of normal GCs and the extreme modifications observed in NGC 2808. Once again, this cluster is a pivotal object to interpret the evidence of multiple populations in GCs.

The discreteness of the different populations detected in NGC 2808 (see Tab. 9) presents another level of challenge. In most scenarios (Gratton Carretta, see) the observed abundance pattern only may be approximated by diluting ejecta of favourite candidate polluters with not processed, primordial matter. It seems difficult to explain populations with discrete composition by imaging a formation history where bursts of star formation are followed by multiple quiescent periods, whereas at the same time the abundances continue to change from a population to the next, following an empirical dilution model. To the difficulty of accounting for the origin and collection of pristine gas (e.g. D'Ercole et al. 2011), one is forced to add mechanisms to selectively stop the mixing and/or star formation several times in a few $10^{7}-10^{8}$ yr at the cluster formation.

A more definitive answer to the questions aroused by these recent discoveries in NGC 2808 must necessarily await when photometric catalogues will be published and the analysis of spectroscopic observations of stars in the photometric groups completed by Milone, Marino and coworkers. Our group just gathered high resolution, high $\mathrm{S} / \mathrm{N}$ spectra for about 100 giants in the present sample to measure $\mathrm{Al}$ abundances, already proved in NGC 6752 (Carretta et al. 2012) and NGC 2808 (Carretta 2014) to be very useful to chemical tag discrete stellar populations on the RGB. With the full set of elements involved in proton-capture reactions we will be able to perform a statistically sound cluster analysis and hopefully disclose other windows on this very particular globular cluster.

We thank Angela Bragaglia for valuable help and discussions and Alessio Mucciarelli for providing his results for Potassium. This publication makes use of data products from the Two Micron All Sky Survey, which is a joint project of the University of Massachusetts and the Infrared Processing and Analysis Center/California Institute of Technology, funded by the National Aeronautics and Space Administration and the National Science Foundation. This research has been funded by PRIN MIUR 2010-2011, project "The Chemical and Dynamical Evolution of the Milky Way and Local Group Galaxies" (PI F. Matteucci). This research has made use of the SIMBAD database, operated at CDS, Strasbourg, France and of NASA's Astrophysical Data System.
Alonso, A., Arribas, S., Martinez-Roger, C. 1999, A\&AS, 140, 261 Alonso, A., Arribas, S., Martinez-Roger, C. 2001, A\&A, 376, 1039 Arnould, M., Goriely, S., Jorissen, A. 1999, A\&A, 347, 572

Bastian, N., Cabrera-Ziri, I., Salaris, M. 2015, MNRAS, 449, 3333

Bedin, L.R., Piotto, G., Zoccali, M. et al. 2000, A\&A, 363, 159

Bragaglia, A., Carretta, E., Gratton, R.G. et al. 2001, AJ, 121, 327

Bragaglia, A., Carretta, E., Gratton, R.G., D’Orazi, V., Cassisi,

S., Lucatello, S. 2010, A\&A, 519, 60

Carretta, E. 2006, AJ, 131, 1766

Carretta, E. 2014, ApJ, 795, L28

Carretta, E., Bragaglia, A., Gratton R.G., Leone, F.,

Recio-Blanco, A., Lucatello, S. 2006, A\&A, 450, 523

Carretta, E., Bragaglia, A., Cacciari, C., Rossetti, E. 2003, A\&A, 410,143

Carretta, E., Bragaglia, A., Cacciari 2004a, ApJ, 610, L25

Carretta, E., Recio-Blanco, A., Gratton, R.G., Piotto, G., Bragaglia, A. 2007b, ApJ, 671, L125

Carretta, E., Bragaglia, A., Gratton, R.G., Lucatello, S. Momany, Y. 2007b, A\&A, 464, 927

Carretta, E., Bragaglia, A., Gratton, R.G. et al. 2007c, A\&A, 464, 939

Carretta, E., Bragaglia, A., Gratton, R.G. et al. 2007d, A\&A, 464, 967

Carretta, E., Recio-Blanco, A., Gratton, R.G., Piotto, G., Bragaglia, A. 2007e, ApJ, 671, L125

Carretta, E., Bragaglia, A., Gratton, R.G., Lucatello, S. 2009a, A\&A, 505, 139

Carretta, E., Bragaglia, A., Gratton, R.G. et al. 2009b, A\&A, 505,117

Carretta, E., Bragaglia, A., Gratton, R.G., D'Orazi, V., Lucatello, S. 2009c, A\&A, 508, 695

Carretta, E., Bragaglia, A., Gratton, R.G., Recio-Blanco, A., Lucatello, S., D'Orazi, V., Cassisi, S. 2010a, A\&A, 516, 55

Carretta, E., Bragaglia, A., Gratton, R.G. et al. 2010b, A\&A, 520,95

Carretta, E., Bragaglia, A., Gratton, R., Lucatello, S., Bellazzini, M., D'Orazi, V. 2010c, ApJ, 712, L21

Carretta, E., Lucatello, S., Gratton, R.G., Bragaglia, A., D’Orazi, V. 2011, A\&A, 533, 69

Carretta, E., Bragaglia, A., Gratton, R.G., Lucatello, S., D’Orazi, V. 2012, ApJ, 750, L14

Carretta, E., Bragaglia, A., Gratton, R.G. et al. 2013, A\&A, 557, A138

Carretta, E., Gratton, R.G., Bragaglia, A., D'Orazi, V., Lucatello, S., Sollima, A., Sneden, C. 2013b, ApJ, 769, 40

Carretta, E., Bragaglia, A., Gratton, R.G. et al. 2014b, A\&A, 564, A60

Carretta, E., Bragaglia, A., Gratton, R.G. et al. 2015, A\&A, 578, A116

Cohen, J.G., Kirby, E.N. 2012, ApJ, 760, 86

Dalessandro, E., Salaris, M., Ferraro, F.R., Cassisi, S., Lanzoni, B., Rood, R.T., Fusi Pecci, F., Sabbi, E. 2011, MNRAS, 410, 694

D'Antona, F., Bellazzini, M., Caloi, V., Fusi Pecci, F., Galleti, S., Rood, R.T. 2005, ApJ, 631, 868

Decressin, T., Meynet, G., Charbonnel C. Prantzos, N., Ekstrom, S. 2007, A\&A, 464, 1029

Denisenkov, P.A., Denisenkova, S.N. 1989, A.Tsir., 1538, 11

D'Ercole, A., D'Antona, F., Vesperini, E. 2011, MNRAS, 415, 1304

Gratton, R.G., Sneden, C., Carretta, E., Bragaglia, A. 2000, A\&A, 354, 169

Gratton, R.G. 1988, Rome Obs. Preprint Ser., 29

Gratton, R.G., Carretta, E., Eriksson, K., Gustafsson, B. 1999, A\&A, 350, 955

Gratton, R.G., Bonifacio, P., Bragaglia, A., et al. 2001, A\&A, 369,87

Gratton, R.G., Carretta, E., Claudi, R., Lucatello, S., \& Barbieri, M. 2003, A\&A, 404, 187

Gratton, R.G., Lucatello, S., Bragaglia, A., Carretta, E.,

Momany, Y., Pancino, E., Valenti, E. 2006, A\&A, 455, 271

Gratton, R.G., Lucatello, S., Bragaglia, A. et al. 2007, A\&A, 464, 953

Gratton, R.G., Lucatello, S., Carretta, E., Bragaglia, A., D’Orazi, V., Momany, Y. 2011, A\&A, 534, 123

Gratton, R.G., Carretta, E., Bragaglia, A. 2012b, A\&ARv, 20, 50 
Grundahl, F., VandenBerg, D.A., Andersen, M.I. 1998, ApJ, 500, L179

Han, S.-I., Lee, Y.-W., Joo, S.-J., Sohn, S.T., Yoon, S.-J., Kim, H.S., Lee, J.-W. 2009, ApJ, 707, L190

Harris, W. E. 1996, AJ, 112, 1487

Iannicola, G., Monelli, M., Bono, G. et al. 2009, ApJ, 696, L120

Karakas, A.I., Lattanzio, J..C. 2003, PASA, 20, 279

Kraft, R.P. 1994, PASP, 106, 553

Kurucz, R.L. 1993, CD-ROM 13, Smithsonian Astrophysical Observatory, Cambridge

Langer, G.E., Hoffman, R., Sneden, C. 1993, PASP, 105, 301

Lee, J.-W., Lee, J., Kang, Y.-W., Lee, Y.-W., Han, S.-I., Joo, S.-J., Rey, S.-C., Yong, D. 2009, ApJ, 695, L78

Magain, P. 1984, A\&A, 134, 189

Marino, A.F., Villanova, S., Piotto, G., Milone, A.P., Momany, Y., Bedin, L.R., Medling, A.M. 2008, A\&A, 490, 625

Marino, A.F., Milone, A.P., Przybilla, N. et al. 2014, MNRAS, 437, 1609

Milone, A., Piotto, G., Bedin, L. et al. 2012a, ApJ, 744, 58

Milone, A., Piotto, G., Bedin, L. et al. 2012b, A\&A, 537, A77

Milone, A.P., Marino, A.F., Piotto, G. et al. 2015, arXiv:1505.05934

Monelli, M., Milone, A.P., Stetson, P.B. et al. 2013, MNRAS, 431,2126

Mucciarelli, A., Bellazzini, M., Ibata, R., Merle, T., Chapman, S.C., Dalessandro, E., Sollima, A. 2012, MNRAS, 426, 2889

Mucciarelli, A., Bellazzini, M., Merle, T., Plez, B., Dalessandro, E., Ibata, R. 2015, ApJ, 801, 68 (Mu15)
Nataf, D., Gould, A.P., Pinsonneault, M.H. Udalski, A. 2011, ApJ, 766, 77

Pasquini, L. et al. 2002, The Messenger, 110, 1

Piotto, G., Bedin, L., Anderson, J. et al. 2007, ApJL, 661, L53

Piotto, G., Milone, A.P., Bedin, L.R. et al. 2015, AJ, 149, 91

Prantzos, N., Charbonnel, C., Iliadis, C. 2007, A\&A, 179, 190

Recio-Blanco, A., Aparicio, A., Piotto, G., De Angeli, F., Djorgovski, S.G. 2006, A\&A, 452, 875

Skrutskie, M.F. et al. 2006, AJ, 131, 1163

Smith, G.H. 1987, PASP, 99, 67

Sneden, C. 2000, in "The Galactic Halo : From Globular Cluster to Field Stars", Proceedings of the 35th Liege International Astrophysics Colloquium,1999. Eds. A. Noels, P. Magain, D. Caro, E. Jehin, G. Parmentier, and A. A. Thoul. Liege, Belgium, 2000., p.159

Tukey, J.W., Explanatory Data Analysis (Reading: Addison-Wesley).

Ventura, P., D'Antona, F., Di Criscienzo, M., Carini, R., D'Ercole, A., Vesperini, E. 2012, ApJ, 761, L30

Ventura, P., Di Criscienzo, M., Carini, R., D'Antona, F. 2013, MNRAS, 431, 3642

Yong, D., Grundahl, F., Nissen, P.E., Jensen, H.R., Lambert, D.L. 2005, A\&A, 438, 875 
Table 1

Adopted atmospheric parameters and derived iron abundances in NGC 2808. The prefix U indicates stars observed with UVES. The complete table is available electronically only at CDS.

\begin{tabular}{rcccccccccc}
\hline Star & $\begin{array}{c}T_{\text {eff }} \\
(\mathrm{K})\end{array}$ & $\begin{array}{c}\log g \\
(\mathrm{dex})\end{array}$ & $\begin{array}{c}{[\mathrm{A} / \mathrm{H}]} \\
(\mathrm{dex})\end{array}$ & $\begin{array}{c}v_{t} \\
\left(\mathrm{~km} \mathrm{~s}^{-1}\right)\end{array}$ & $\begin{array}{c}{[\mathrm{Fe} / \mathrm{H}] \mathrm{I}} \\
(\mathrm{dex})\end{array}$ & $\begin{aligned} r m s \\
\mathrm{n}\end{aligned}$ & $\begin{array}{r}{[\mathrm{Fe} / \mathrm{HII}} \\
(\mathrm{dex})\end{array}$ & $r m s$ \\
\hline 7183 & 4531 & 1.52 & -1.08 & 1.70 & 46 & -1.080 & 0.071 & 4 & -1.121 & 0.257 \\
7315 & 4459 & 1.45 & -1.14 & 1.29 & 54 & -1.138 & 0.086 & 3 & -1.075 & 0.078 \\
$\mathrm{U} 7536$ & 4306 & 1.18 & -1.11 & 1.55 & 89 & -1.109 & 0.079 & 10 & -1.125 & 0.055 \\
7558 & 4711 & 1.87 & -1.13 & 1.64 & 47 & -1.129 & 0.086 & 4 & -1.106 & 0.270 \\
7788 & 4510 & 1.53 & -1.15 & 1.44 & 20 & -1.144 & 0.058 & & & \\
\hline
\end{tabular}

Note - This table is available in its entirety in a machine-readable form in the online journal. A few lines are shown here for guidance regarding its form and content.

Table 4

Abundances of proton-capture elements in stars of NGC 2808. Upper limits (limO=0)and detections $(=1)$ for $\mathrm{O}$ are flagged. The complete table is available electronically only at CDS.

\begin{tabular}{rccccccccccccc}
\hline star & $\mathrm{n}$ & {$[\mathrm{O} / \mathrm{Fe}]$} & $\mathrm{rms}$ & $\mathrm{n}$ & {$[\mathrm{Na} / \mathrm{Fe}]$} & $\mathrm{rms}$ & $\mathrm{n}$ & {$[\mathrm{Mg} / \mathrm{Fe}]$} & $\mathrm{rms}$ & $\mathrm{n}$ & {$[\mathrm{Si} / \mathrm{Fe}]$} & $\mathrm{rms}$ & $\operatorname{limO}$ \\
\hline 7183 & 2 & +0.232 & 0.033 & 4 & +0.135 & 0.023 & 3 & +0.323 & 0.077 & 10 & +0.261 & 0.084 & 1 \\
7315 & 2 & +0.352 & 0.047 & 4 & -0.150 & 0.040 & 3 & +0.363 & 0.101 & 12 & +0.278 & 0.085 & 1 \\
$\mathrm{U} 7536$ & 2 & +0.248 & 0.040 & 4 & +0.060 & 0.017 & 3 & +0.355 & 0.095 & 8 & +0.260 & 0.039 & 1 \\
7558 & 1 & +0.392 & & 3 & -0.076 & 0.067 & 2 & +0.370 & 0.009 & 11 & +0.249 & 0.072 & 1 \\
7788 & 0 & & & 2 & +0.224 & 0.026 & 1 & +0.352 & & 8 & +0.236 & 0.066 & 1 \\
\hline
\end{tabular}

Note - This table is available in its entirety in a machine-readable form in the online journal. A few lines are shown here for guidance regarding its form and content.

Table 5

Abundances of $\alpha$ - and Fe-peak elements in stars of NGC 2808. The complete table is available electronically only at CDS.

\begin{tabular}{|c|c|c|c|c|c|c|c|c|c|c|c|c|c|c|c|}
\hline star & $\mathrm{n}$ & {$[\mathrm{Ca} / \mathrm{Fe}]$} & rms & $\mathrm{n}$ & {$[\mathrm{Ti} / \mathrm{Fe}] \mathrm{I}$} & $\mathrm{rms}$ & $\mathrm{n}$ & {$[\mathrm{Sc} / \mathrm{Fe}]$ II } & $\mathrm{rms}$ & $\mathrm{n}$ & {$[\mathrm{Cr} / \mathrm{Fe}] \mathrm{I}$} & rms & $\mathrm{n}$ & {$[\mathrm{Ni} / \mathrm{Fe}]$} & $\mathrm{rms}$ \\
\hline 7183 & 6 & 326 & 059 & 5 & +0.228 & 0.071 & 6 & -0.066 & 0.071 & 5 & -0.052 & 0.078 & 11 & -0.1 & .087 \\
\hline 7315 & 7 & 0.317 & 0.1 & 5 & +0.159 & 078 & 6 & +0.039 & 0.151 & 3 & 53 & 8 & 7 & 3 & 0.031 \\
\hline U7536 & 13 & +0.318 & 0.069 & 10 & 39 & 0.047 & 8 & -0.045 & 3 & 18 & 51 & 39 & 30 & -( & .064 \\
\hline 7558 & 7 & +0.332 & 0.102 & 6 & +0.215 & 0.072 & 6 & -0.057 & 0.072 & 5 & -0.061 & 0.081 & 10 & -0.061 & 0.219 \\
\hline 7788 & 1 & +0.339 & & 2 & +0.236 & 0.042 & 5 & +0.050 & 0.062 & 5 & -0.024 & 0.121 & 2 & -0.064 & 0.007 \\
\hline
\end{tabular}

Note - This table is available in its entirety in a machine-readable form in the online journal. A few lines are shown here for guidance regarding its form and content.

Table 6

Abundances of Ti II, Cr II, and Mn in stars of NGC 2808 observed with UVES.

\begin{tabular}{rccccccccc}
\hline star & $\mathrm{n}$ & {$[\mathrm{Ti} / \mathrm{Fe}] \mathrm{II}$} & $\mathrm{rms}$ & $\mathrm{n}$ & {$[\mathrm{Cr} / \mathrm{Fe}] \mathrm{II}$} & $\mathrm{rms}$ & $\mathrm{n}$ & {$[\mathrm{Mn} / \mathrm{Fe}]$} & $\mathrm{rms}$ \\
\hline 8739 & 9 & +0.208 & 0.094 & 14 & -0.011 & 0.118 & 3 & -0.395 & 0.026 \\
38660 & 8 & +0.186 & 0.110 & 17 & -0.048 & 0.174 & 3 & -0.355 & 0.009 \\
8603 & 9 & +0.195 & 0.091 & 14 & -0.071 & 0.120 & 3 & -0.378 & 0.080 \\
10571 & 8 & +0.197 & 0.072 & 12 & -0.020 & 0.067 & 3 & -0.399 & 0.048 \\
30763 & 9 & +0.200 & 0.070 & 14 & -0.034 & 0.133 & 3 & -0.359 & 0.066 \\
\hline
\end{tabular}

Note - This table is available in its entirety in a machine-readable form in the online journal. A few lines are shown here for guidance regarding its form and content. 